\title{
Impact of Voluntary Disclosure on the Relevance of Accounting Information
}

\author{
*Jihene Chedlia Soussi, Mounira Ben Arab \\ ISG Tunis \\ *jihenesoussi@yahoo.com
}

\begin{abstract}
Information disclosure by firms has grown considerably. The increased level of firms' disclosure has been accompanied by the loss of relevance of accounting information over time (Lev, 1989, Ramech and Thiagarajan, 1995, Lev and Zarowin, 1999, Brown and al, 1999, Chang, 1999 and Chalmers and al, 2011). Our objective is to determine whether the voluntary disclosure explains the low relevance of accounting information. We find that medium-technology companies have the highest level of relevance of accounting information. However, the relevance of the accounting model is low for lowtechnology firms and high technology firms. The introduction of the overall disclosure index and subindexes of disclosure has an effect on the relevance of the accounting model (this effect is significant only in some cases for low-tech firms). Furthermore, the addition of variables of disclosure to the accounting model makes the accounting variables relevant to investors for low-tech firms. For medium-tech firms, book values and earnings are relevant. While, for high technology firms, only the earnings are relevant. We also show that the introduction of intangible expenses, the weight of intangibles and the index of disclosure on intangibles is growing, but not significantly the relevance of the accounting model.
\end{abstract}

Keywords: Value relevance, Accounting information, Voluntary Disclosure, intangibles, disclosure index

\section{Introduction}

Information disclosure by firms has grown considerably. Indeed, firms disclose more and more nonfinancial information through formal or informal means. The increased level of firms' disclosure has been accompanied by a loss of accounting information's relevance over time. The latter finding was demonstrated by Lev and Zarowin (1999), Brown and al (1999), Chang (1999), Soussi, Matoussi and Mouelhi (2006) and Chalmers and al (2011). Collins and al. (1997) find that the loss of accounting information's relevance is explained by intangible investments, the frequency of non-recurring items and frequency of losses. Lev and Zarowin (1999) showed that losses and nonrecurring items are not causes, but these are symptoms of the decrease of accounting information's relevance. Soussi, Matoussi and Mouelhi (2006) show that intangible investments and losses explain partly the low relevance of accounting information. Amir and Lev (1996) show that accounting information is relevant only when used in conjunction with non-financial information. Thus, growth in the level of voluntary disclosure is a main factor explaining the decline of accounting information's relevance (Lundohlm et al, 2000). Our research is part of current research to explain the low level of relevance of accounting information. Our objective is to determine whether the voluntary disclosure explains the low relevance of accounting information. In the first part, we investigate whether the level of disclosure (measured by the overall disclosure) explains this decline of relevance. In the second part, we check if the disclosure level operationalized by the disclosure on intangibles explains this low relevance. We use sub-indexes of disclosure for each category of information and we study the effect of each of these sub-indexes on the relevance of accounting information to determine the categories of information that lead to low relevance of accounting information. We study the Canadian market because the Canadian standards are quite similar to IFRS standards that are currently the most encouraged ${ }^{1}$ and the Canadian model is representative of the Anglo American market model in which there is a significant pressure to communicate information to investors because corporate financing is done mostly through the capital market. $^{2}$

\section{Literature Review}

\footnotetext{
${ }^{1}$ According to a document published by the Canadian Accounting Standards Board entitled "Comparison of Canadian GAAP with IFRS," based on standards published in March 31, 2005.

${ }^{2}$ Ding, and al 2004.
} 
Amir and Lev (1996) show that earnings and book values are significantly associated with prices and returns when used in conjunction with non-financial information. Therefore, the non-financial information reduces the relevance of accounting information. Lee and al (2005) attribute the low correlation between stock returns and earnings mainly to noise resulting from the uncertainty. They add that to reduce the uncertainty associated with future earnings, the high-tech firms could provide investors with additional information. Lundholm et al (2002) show that the stock returns of firms better reflect future earnings when their level of voluntary disclosure increases and so the voluntary disclosure decreases the relevance of accounting information (same result found by Luo and al, 2006). Dontoh et al (2004) show that the decline of the association between security prices and accounting information is caused by the increase of non- information based negotiations. In Brazil, Lopes (2003) show that accounting numbers are more relevant for high-tech firms than for traditional firms. He explains his results by the fact that in the emerging market that he studied, the sources of information are less available than in developed markets. Unlike the above-mentioned articles, Healy et al (1999) find that firms with increasing levels of disclosure have also increasing relevance of current earnings. Lundohlm et al. (2000) have explained the contradiction with their results by the fact that since Healy and al (1999) did not use future earnings in the regression, the coefficients of current earnings necessarily capture the change in forecast future earnings. Also, Zhao (2002) shows that the level of disclosure about research and development has a significant effect on the relevance of accounting information. Furthermore, Gelb and Zarowin (2002) find that firms with high rates of disclosure have a higher degree of association between stock prices on the one hand and current earnings and future earnings on the other hand, relative to firms with low rates of disclosure. The results obtained by Lapointe-Antunes et al (2006) show that Swiss firms use discretionary accruals to smooth earnings. But this phenomenon is reduced for firms that voluntarily disclose more information in their annual reports or applying IFRS or GAAP. However, Banghøj and Plenborg (2008) find that a higher level of voluntary disclosure does not improve the association between current returns and future earnings. They ask the question: does the voluntary disclosure contains relevant information on future earnings or is that investors are unable to incorporate the voluntary information in the estimates of the firms' values?

\section{Methodology}

According to our literature review, we propose to test the following hypotheses:

* Hypothesis 1: The firm's level of disclosure affects the relevance of the accounting model for evaluating companies

* Hypothesis 2: The level of disclosure affects the relevance of accounting numbers of firms.

Sample and Data Collection: We study a random sample of Canadian firms from the database found at www. SEDAR.com. We exclude firms in the banking and insurance sector to avoid the heterogeneity of accounting practices. Data is collected from annual reports for 2006 and 2007 (54 firms). We use www. finance.yahoo.com to collect information on market prices of corporate stocks. The analysis is performed on the total sample, then by breaking the sample into three subsamples: low-technology companies, medium-tech companies and high-tech companies.

Specification of models: We estimate regression models in which the market value of the company is the dependent variable and earnings and the book value of equity are independent variables (Ohlson, 1995, Collins and al, 1997, Barth and al, 1998). According to Brown and al (1999), researches operationalize relevance by the $\mathrm{R}^{2}$ of the regressions of stock prices on earnings per share and book value of equity per share. Inter-temporal differences and cross-sample differences of $\mathrm{R}^{2}$ are used as indicators that the relevance of accounting information has changed over time or that the relevance differs across disclosure regimes. Brown and al. (1999) estimate that the price model based on earnings and book value used by Collins and al (1997) and other researches, does not take into account the scale effect. Therefore, these variables must be adjusted by the price beginning of year as recommended by Christie (1987), Brown and al (1999) and Lo (2004). Lev and Zarowin (1999) consider a level of $61.8 \%$ of $\mathrm{R}^{2}$ for the model of the price based on book values and earnings as low level of $\mathrm{R}^{2}$.

-Dependent Variable: Market value of the firm: it is the stock value 3 months after the end of the fiscal year (Collins and al, 1997, Ohlson, 1995).

-Independent Variables: Book value of equity of the firm: it is the value of equity that are the property of shareholders (Allen, 2003). Using the number of shares = average number of shares outstanding, we 
have: Book value of the company = (total assets at the end of the fiscal year - total liabilities at the end of the fiscal year) / weighted average number of shares outstanding.

Net income before extraordinary items: Lev and Sougiannis (1996) used earnings before extraordinary items (also Lev and Zarowin, 1999).

Level of Disclosure: Botosan (1997) uses the annual report as the base of his disclosure index because the annual report is generally considered one of the most important information about firms. Furthermore, the level of disclosure in annual reports is positively correlated with the level of information provided by other information mediums (Lang and Lundholm, 1993). Trabelsi and Labelle (2006) classify the content of financial reporting in seven categories of voluntary disclosure (see Appendix 4) useful to investors, financial analysts and standard setters. We use the same index of disclosure of Trabelsi and Labelle (2006) who study Canadian firms. Al-Shammari and Al-Sultan (2010) calculate the index of voluntary disclosure as the ratio of total items actually disclosed divided by the maximum possible score of items. Following this research, we use this method.

-Control variables: They are: Size (Log TA), growth rate (GR), financial profitability (FP) and Leverage (Lev).The market value is positively associated with firm size, growth rate, financial profitability and Leverage (Cazavan-Jeny, 2004).

Holthausen and Watts (2001) and Aboody and Lev (1998) show that managers, at the capitalization of certain intangibles, skew the amounts recognized for contractual reasons such that contract terms on the debt.

Multiple regressions: In our research we estimate the models that are detailed in the following:

Model 1: Basic model

$\frac{P_{i, t}}{P_{i, t-1}}=\alpha_{0}+\alpha_{1} \frac{E_{i, t}}{P_{i, t-1}}+\alpha_{2} \frac{B V_{i, t}}{P_{i, t-1}}+\varepsilon_{i, t}(1)$

$\varepsilon i, \mathrm{t}=$ residue.

i: i company.

$\mathrm{t}$ : year $(\mathrm{t}=1$ for $2006, \mathrm{t}=2$ for 2007).

$\mathrm{P}=$ Share price of the company three months after the end of the fiscal year.

$\mathrm{BV}=$ compagny's book value of equity at the end of the fiscal year divided by the number of shares.

$\mathrm{E}=$ net income before extraordinary items per share of the company.

Model 2 : Basic model with the introduction of control variables

$P_{i, t}=a_{0}+a_{1} E_{i, t}+a_{2} B V_{i, t}+a_{3} \log T A_{i, t}+a_{4} G_{i, t}+a_{5} F_{i, t}+a_{6} \operatorname{Lev}_{i, t}+\varepsilon_{i, t}$.

$\mathbf{P}_{\mathrm{i}, \mathrm{t}-1} \quad \stackrel{\mathbf{P}_{\mathrm{i}, \mathrm{t}-1}}{\mathbf{P}_{\mathrm{i}, \mathrm{t}-1}}$

$\log \mathrm{TA}=\log$ of total assets ( $\log$ base 10$)$.

$\mathrm{GR}=$ growth rate $=$ variation in turnover between $\mathrm{t}$ and $\mathrm{t}-1$

$\mathrm{FP} \quad=$ financial profitability or return=net income /equity.

Lev = Leverage=long-term debt/equity.

Model 3 : Basic Model with the introduction of control variables and index of global disclosure

$\underline{P_{i, t}}=b_{0}+b_{1} E_{i, t}+b_{2} B V_{i, t}+b_{3} D_{i, t}+b_{4} \operatorname{LogT} A_{i, t}+b_{5} G_{i, t}+b_{6} F_{i, t}+b_{7} \operatorname{LeV}_{i, t}+\varepsilon_{i, t}$.

$\begin{array}{lll}\mathbf{P}_{\mathrm{i}, \mathrm{t}-1} & \mathbf{P}_{\mathrm{i}, \mathrm{t}-1} & \mathbf{\mathbf { P } _ { \mathrm { i } , \mathrm { t } - 1 }}\end{array}$

$\mathrm{Dg}_{\mathrm{i}, \mathrm{t}}=$ Index of overall disclosure

Model 4 : Basic Model with the introduction of control variables and D1

$P_{i, t}=b_{0}+b_{1} E_{i, t}+b_{2} B V_{i, t}+b_{3} D 1_{i, t}+b_{4} \operatorname{LogTA}_{i, t}+b_{5} G_{i, t}+b_{6} F_{i, t}+b_{7} \operatorname{LeV}_{i, t}+\varepsilon_{i, t}$.

$\mathbf{P}_{\mathrm{i}, \mathrm{t}-1} \quad \mathbf{P}_{\mathrm{i}, \mathrm{t}-1} \quad \mathbf{P}_{\mathrm{i}, \mathrm{t}-\mathrm{t}}$

We replace D1 by D2, D3, D4, D5, D6 and D7and we study each model obtained.

D1: index of background information disclosure, D2: index of disclosure of summary of past results, D3: index of disclosure of non- financial key statistics, D4:disclosure index of forecast information, D5: index of disclosure of discussion and analysis of management, D6: Index of disclosure on intangible assets, D7: index of disclosure of social and environmental information. 
Classification by sector: We test the stability of models between the three sub-samples ${ }^{3}$ through the Chow test. We use STATA which is suitable for the treatment of panel data.

\section{Results and Discussion of the first part}

Table 1: Results for basic model (model 1) and basic model with introduction of control variables

\begin{tabular}{|c|c|c|c|c|c|c|c|c|}
\hline Model & Basic mode & & & & & & & \\
\hline $\begin{array}{l}\text { Group } \\
\text { variable }\end{array}$ & $\begin{array}{l}\text { Total } \\
\text { Sample }\end{array}$ & $\begin{array}{l}\text { Low } \\
\text { technology }\end{array}$ & $\begin{array}{l}\text { Medium } \\
\text { technology }\end{array}$ & $\begin{array}{l}\text { High } \\
\text { technology }\end{array}$ & $\begin{array}{l}\text { Total } \\
\text { sample }\end{array}$ & $\begin{array}{l}\text { Low } \\
\text { technology }\end{array}$ & $\begin{array}{l}\text { Medium } \\
\text { technology }\end{array}$ & $\begin{array}{l}\text { High } \\
\text { Technology }\end{array}$ \\
\hline$B V_{i, t} / P_{i, t-1}$ & $\begin{array}{l}0.883 \\
(45.38)^{* * *}\end{array}$ & $\begin{array}{l}0.498 \\
(1.72)\end{array}$ & $\begin{array}{l}0.873 \\
(39.37)^{* * *}\end{array}$ & $\begin{array}{l}-0.205 \\
(-0.39)\end{array}$ & $\begin{array}{l}0.826 \\
(43.32)^{* * *}\end{array}$ & & $\begin{array}{l}0.814 \\
(38.35)^{* * *}\end{array}$ & $\begin{array}{l}-0.229 \\
(-0.44)\end{array}$ \\
\hline $\mathrm{E}_{\mathrm{i}, \mathrm{t}} / \mathrm{P}_{\mathrm{i}, \mathrm{t}-1}$ & $\begin{array}{l}-1.725 \\
(-7.09)^{* * *}\end{array}$ & $\begin{array}{l}1.549 \\
(0.99)\end{array}$ & $\begin{array}{l}-1.904 \\
(-6.78)^{* * *}\end{array}$ & $\begin{array}{l}3.201 \\
(3.14)^{* * *}\end{array}$ & $\begin{array}{l}-2.551 \\
(-10.39)^{* * *}\end{array}$ & & $\begin{array}{l}-2.747 \\
(-9.90)^{* * *}\end{array}$ & $\begin{array}{l}6.191 \\
(2.87)^{* * *}\end{array}$ \\
\hline $\log \mathrm{TA}$ & & & & & $\begin{array}{l}-0.299 \\
(-4.76)^{* * *}\end{array}$ & & $\begin{array}{l}-0.303 \\
(-2.50)^{* *}\end{array}$ & $\begin{array}{l}-0.224 \\
(-1.32)\end{array}$ \\
\hline Lev & & & & & $\begin{array}{l}0.306 \\
(1.18)\end{array}$ & & $\begin{array}{l}0.417 \\
(1.30)\end{array}$ & $\begin{array}{l}-0.179 \\
(-0.16)\end{array}$ \\
\hline FP & & & & & $\begin{array}{l}0.325 \\
(5.29)^{* * *}\end{array}$ & & $\begin{array}{l}0.321 \\
(4.66)^{* * *}\end{array}$ & $\begin{array}{l}-0.902 \\
(-1.80)^{*}\end{array}$ \\
\hline GR & & & & & $\begin{array}{l}4.17 E-09 \\
(2.22)^{* *}\end{array}$ & & $\begin{array}{l}5.16 \mathrm{E}-09 \\
(2.17)^{* *}\end{array}$ & $\begin{array}{l}7.51 \mathrm{E}-08 \\
(0.49)\end{array}$ \\
\hline $\begin{array}{l}\text { Hausman } \\
\text { test }\end{array}$ & & & & & & $\begin{array}{l}\text { CHI2= } \\
-38.98 \\
\text { CHI } 2<0\end{array}$ & & \\
\hline $\begin{array}{l}\mathrm{R}^{2} \text { and } \\
\text { number } \\
\text { of } \\
\text { observations }\end{array}$ & $\begin{array}{l}\mathrm{R}^{2}= \\
0.9920 \\
\mathrm{~F} \\
5981.36 \\
\mathrm{P}=0.0000 \\
\mathrm{n}=99\end{array}$ & $\begin{array}{l}R^{2}=0.1742 \\
F=1.90 \\
P=0.1786 \\
n=21\end{array}$ & $\begin{array}{l}R^{2}=0.9954 \\
F=4785.29 \\
P=0.0000 \\
N=47\end{array}$ & $\begin{array}{l}\mathrm{R}^{2}= \\
0.2609 \\
\mathrm{~F}=4.94 \\
\mathrm{P}=0.0145 \\
\mathrm{n}=31\end{array}$ & $\begin{array}{l}\mathrm{R}^{2}= \\
0.9948 \\
\mathrm{~F} \\
2915.21 \\
\mathrm{P}=0.0000 \\
\mathrm{n}=99\end{array}$ & & $\begin{array}{l}R^{2}=0.9973 \\
F=2464.68 \\
P=0.0000 \\
n=47\end{array}$ & $\begin{array}{l}\mathrm{R}^{2}=0.3852 \\
\mathrm{~F}=2.51 \\
\mathrm{P}=0.0502 \\
\mathrm{n}=31\end{array}$ \\
\hline
\end{tabular}

Legend of Tables 1, 2 and 3:

Low technology: LT : low technology sector

Medium technology: MT : medium technology sector.

High technology: HT : high technology sector.

$\mathrm{Pi}, \mathrm{t}=$ Share price of the company three months after the end of the fiscal year.

$\mathrm{BV}=$ book value of equity of the company at the end of the fiscal year divided by the number of shares.

$\mathrm{E}=$ net income before extraordinary items per share of the company

$\operatorname{LogTA}=\log$ of total assets (log base 10).

$\mathrm{GR}=$ Growth rate = change in sales from $\mathrm{t}$ to $\mathrm{t}-1$.

$\mathrm{FP}=$ financial profitability or financial return=net income /equity.

Lev=Leverage=long-term debt/equity.

D1: index of disclosure of general information.

D2: index of disclosure of summary of historical results.

D3: index of disclosure of key non - financial statistics.

D4: index of disclosure of forecast information.

D5: index of disclosure in the Management report.

D6: index of disclosure on intangibles

D7: index of disclosure of social and environmental information

$\mathrm{F}=$ value of the Fisher statistic.

P: probability of $F($ or $\chi 2$ )greater than the calculated critical value of $F(\chi 2)$ or significance of $F($ or $\chi 2)$.

OLS: ordinary least squares

FE: fixed effects

RE: random effects

$\mathrm{R}^{2}$ : model's explanatory power.

n: number of observations.

Values in parentheses are the values of t-statistics.

*Significant at $10 \%$

5 See appendix 2 
** Significant at $5 \%$

***Significant at $1 \%$

Table 2 : Results of multiples regressions (suite)

\begin{tabular}{|c|c|c|c|c|c|c|c|c|c|c|c|c|c|c|c|c|}
\hline \multirow{2}{*}{$\begin{array}{l}\text { Model } \\
\text { Group } \\
\text { variable } \\
\end{array}$} & \multicolumn{4}{|c|}{$\begin{array}{l}\text { Basic model with introduction } \\
\text { of control variables and } D g\end{array}$} & \multicolumn{8}{|c|}{$\begin{array}{l}\text { Basic model with introduction of Basic model with introduction } \\
\text { control variables and D1 of control variables and D2 }\end{array}$} & \multicolumn{4}{|c|}{$\begin{array}{l}\text { Basic model with introduction } \\
\text { of control variables and D3 }\end{array}$} \\
\hline & $\begin{array}{l}\text { Total } \\
\text { sample }\end{array}$ & LT & MT & HT & $\begin{array}{l}\text { Total } \\
\text { sample }\end{array}$ & LT & MT & HT & $\begin{array}{l}\text { Total } \\
\text { sample }\end{array}$ & LT & MT & HT & $\begin{array}{l}\text { Total } \\
\text { sample }\end{array}$ & LT & MT & HT \\
\hline$\overline{B V_{i, t} / P_{i, t-1}}$ & $\begin{array}{l}0.825 \\
(42.91)^{* * *}\end{array}$ & $\begin{array}{ll}-0.357 \\
-(-1.33)\end{array}$ & $\begin{array}{l}0.811 \\
(37.96)^{* *}\end{array}$ & $\begin{array}{c}0.143 \\
(0.20)\end{array}$ & $\begin{array}{l}0.826 \\
(43.06)^{* * *}\end{array}$ & $\begin{array}{lll}0.688 \\
(2.42)^{* *}\end{array}$ & $\begin{array}{l}0.813 \\
(38.11)^{* * *}\end{array}$ & $\begin{array}{l}0.111 \\
*(0.19)\end{array}$ & $\begin{array}{l}0.825 \\
(43.59)^{* * *}\end{array}$ & $\begin{array}{ll}0.759 \\
*(2.75)^{* *}\end{array}$ & $\begin{array}{l}0.813 \\
(38.98)^{* * *}\end{array}$ & $\begin{array}{c}-0.364 \\
*(-0.66)\end{array}$ & $\begin{array}{l}0.826 \\
(43.14)^{* * *}\end{array}$ & $\begin{array}{ll}0.856 \\
*(2.97)^{* *}\end{array}$ & $\begin{array}{l}0.813 \\
(38.13)^{* * *}\end{array}$ & $\begin{array}{c}-0.209 \\
*(-0.39)\end{array}$ \\
\hline $\mathrm{E}_{\mathrm{i}, \mathrm{t}} / \mathrm{P}_{\mathrm{i}, \mathrm{t}-1}$ & $\begin{array}{l}-2.578 \\
(- \\
10.34)^{* * * *}\end{array}$ & $\begin{array}{l}-19.739 \\
(-3.79)^{* *}\end{array}$ & $\begin{array}{l}-2.803 \\
(-9.95)^{* * *}\end{array}$ & $\begin{array}{l}5.228 \\
*(2.08)^{* *}\end{array}$ & $\begin{array}{l}-2.556 \\
(- \\
10.34)^{* * *}\end{array}$ & $\begin{array}{l}5.746 \\
(1.49)\end{array}$ & $\begin{array}{l}-2.756 \\
(-9.88)^{* * * *}\end{array}$ & $\begin{array}{l}4.671 \\
(1.92)^{*}\end{array}$ & $\begin{array}{l}-2.597 \\
(- \\
10.60)^{* * *}\end{array}$ & $\begin{array}{l}1.932 \\
(0.47)\end{array}$ & $\begin{array}{l}-2.780 \\
(- \\
10.16)^{* * *}\end{array}$ & $\begin{array}{l}6.50 \\
(2.95)^{* * *}\end{array}$ & $\begin{array}{l}-2.555 \\
*(- \\
10.36)^{* * *}\end{array}$ & $\begin{array}{l}1.742 \\
(0.42)\end{array}$ & $\begin{array}{l}-2.752 \\
(-9.87)^{* * *}\end{array}$ & $\begin{array}{l}5.867 \\
(2.43)^{* *}\end{array}$ \\
\hline Dg & $\begin{array}{l}0.816 \\
(0.69)\end{array}$ & $\begin{array}{l}11.784 \\
(2.16)\end{array}$ & $\begin{array}{l}2.167 \\
(1.08)\end{array}$ & $\begin{array}{l}-3.214 \\
(-0.77)\end{array}$ & & & & & & & & & & & & \\
\hline D1 & & & & & $\begin{array}{l}0.237 \\
(0.35)\end{array}$ & $\begin{array}{l}-2.567 \\
(-1.37)\end{array}$ & $\begin{array}{l}0.933 \\
(0.81)\end{array}$ & $\begin{array}{l}-2.498 \\
(-1.29)\end{array}$ & & & & & & & & \\
\hline D2 & & & & & & & & & $\begin{array}{l}0.772 \\
(1.62)\end{array}$ & $\begin{array}{l}1.462 \\
(1.78)^{*}\end{array}$ & $\begin{array}{l}1.077 \\
(1.54)\end{array}$ & $\begin{array}{l}0.732 \\
(0.83)\end{array}$ & & & & \\
\hline D3 & & & & & & & & & & & & & $\begin{array}{l}0.646 \\
(0.54)\end{array}$ & $\begin{array}{l}10.293 \\
(1.81)^{*}\end{array}$ & $\begin{array}{l}1.785 \\
(0.80)\end{array}$ & $\begin{array}{l}-0.762 \\
(-0.32)\end{array}$ \\
\hline $\log \mathrm{TA}$ & $\begin{array}{l}-0.286 \\
(-4.35)^{* * *}\end{array}$ & $\begin{array}{l}-1.162 \\
*(-1.33)\end{array}$ & $\begin{array}{l}-0.295 \\
(-2.44)^{* *}\end{array}$ & $\begin{array}{l}-0.200 \\
(-1.16)\end{array}$ & $\begin{array}{l}-0.292 \\
(-4.41)^{* * *}\end{array}$ & $\begin{array}{l}-0.102 \\
(-0.23)\end{array}$ & $\begin{array}{l}-0.293 \\
(-2.40)^{* *}\end{array}$ & $\begin{array}{l}-0.187 \\
(-1.11)\end{array}$ & $\begin{array}{l}-0.312 \\
(-4.97)^{* * *}\end{array}$ & $\begin{array}{l}-0.661 \\
(-2.21)^{* *}\end{array}$ & $\begin{array}{l}-0.283 \\
*(-2.36)^{* *}\end{array}$ & $\begin{array}{l}-0.231 \\
(-1.36)\end{array}$ & $\begin{array}{l}-0.288 \\
(-4.35)^{* * *}\end{array}$ & $\begin{array}{c}-0.487 \\
*(-1.66)\end{array}$ & $\begin{array}{l}-0.296 \\
(-2.42)^{* *}\end{array}$ & $\begin{array}{l}-0.204 \\
(-1.12)\end{array}$ \\
\hline Lev & $\begin{array}{l}0.251 \\
(0.92)\end{array}$ & $\begin{array}{l}2.721 \\
(3.00)^{*}\end{array}$ & $\begin{array}{l}0.258 \\
(0.73)\end{array}$ & $\begin{array}{l}0.883 \\
(0.49)\end{array}$ & $\begin{array}{l}0.277 \\
(1.02)\end{array}$ & $\begin{array}{l}-0.861 \\
(-0.95)\end{array}$ & $\begin{array}{l}0.275 \\
(0.75)\end{array}$ & $\begin{array}{l}0.924 \\
(0.65)\end{array}$ & $\begin{array}{l}0.212 \\
(0.81)\end{array}$ & $\begin{array}{l}0.071 \\
(0.11)\end{array}$ & $\begin{array}{l}0.325 \\
(1.01)\end{array}$ & $\begin{array}{l}-0.461 \\
(-0.38)\end{array}$ & $\begin{array}{l}0.286 \\
(1.09)\end{array}$ & $\begin{array}{l}-0.621 \\
(-0.85)\end{array}$ & $\begin{array}{l}0.380 \\
(1.16)\end{array}$ & $\begin{array}{l}0.177 \\
(0.11)\end{array}$ \\
\hline FP & $\begin{array}{l}0.322 \\
(5.20)^{* * *}\end{array}$ & $\begin{array}{l}29.231 \\
(4.99)^{* *}\end{array}$ & $\begin{array}{l}0.316 \\
(4.58)^{* * *}\end{array}$ & $\begin{array}{l}-0.702 \\
(-1.23)\end{array}$ & $\begin{array}{l}0.322 \\
(5.17)^{* * *}\end{array}$ & $\begin{array}{l}1.774 \\
(0.62)\end{array}$ & $\begin{array}{l}0.311 \\
(4.43)^{* * *}\end{array}$ & $\begin{array}{l}-0.629 \\
(-1.17)\end{array}$ & $\begin{array}{l}0.321 \\
(5.26)^{* * *}\end{array}$ & $\begin{array}{l}1.629 \\
(0.64)\end{array}$ & $\begin{array}{l}0.310 \\
(4.55)^{* * * *}\end{array}$ & $\begin{array}{l}-0.946 \\
(-1.87)^{*}\end{array}$ & $\begin{array}{l}0.322 \\
(5.18)^{* * * *}\end{array}$ & $\begin{array}{l}0.744 \\
(0.32)\end{array}$ & $\begin{array}{l}0.317 \\
(4.56)^{* * *}\end{array}$ & $\begin{array}{l}-0.805 \\
(-1.36)\end{array}$ \\
\hline GR & $\begin{array}{l}4.22 \mathrm{E}-09 \\
(2.24)^{* *}\end{array}$ & $\begin{array}{l}4.28 \mathrm{E}-09 \\
(0.95)\end{array}$ & $\begin{array}{l}5.30 \mathrm{E}-09 \\
(2.23)^{* *}\end{array}$ & $\begin{array}{l}1.09 \mathrm{E}-07 \\
(0.67)\end{array}$ & $\begin{array}{l}\text { 4.14E-09 } \\
(2.19)^{* *}\end{array}$ & $\begin{array}{l}1.74 \mathrm{E}-09 \\
(0.40)\end{array}$ & $\begin{array}{l}5.02 \mathrm{E}-09 \\
(2.10)^{* *}\end{array}$ & $\begin{array}{l}1.60 \mathrm{E}-07 \\
(0.97)\end{array}$ & $\begin{array}{c}74.46 \mathrm{E}-09 \\
(2.39)^{* *}\end{array}$ & $\begin{array}{l}2.08 \mathrm{E}-09 \\
(0.51)\end{array}$ & $\begin{array}{c}95.14 \mathrm{E}-09 \\
(2.21)^{* *}\end{array}$ & $\begin{array}{l}4.51 \mathrm{E}-08 \\
(0.28)\end{array}$ & $\begin{array}{c}84.20 \mathrm{E}-09 \\
(2.23)^{* *}\end{array}$ & $\begin{array}{l}-6.62 \mathrm{E}- \\
10 \\
(-0.14)\end{array}$ & $\begin{array}{l}5.26 \mathrm{E}-09 \\
(2.20)^{* *}\end{array}$ & $\begin{array}{l}9.18 \mathrm{E}- \\
08 \\
(0.55)\end{array}$ \\
\hline $\begin{array}{l}\text { Test of } \\
\text { homogeneity }\end{array}$ & $\begin{aligned} \mathrm{F} & =1.04 \\
\mathrm{yP} & =0.4569\end{aligned}$ & $\begin{aligned} F & =12.77 \\
9 & =0.0297\end{aligned}$ & $\begin{aligned} F & =2.19 \\
P & =0.0595\end{aligned}$ & $\begin{aligned} F & =1.42 \\
5 \mathrm{P} & =0.3480\end{aligned}$ & $\begin{array}{rr}\mathrm{F} & =1.06 \\
\mathrm{P} & =0.4326\end{array}$ & $\begin{array}{l}6 \mathrm{~F}=5.00 \\
6 \mathrm{P}=0.106\end{array}$ & $\begin{aligned} \mathrm{F} & =1.90 \\
0 \mathrm{P} & =0.1001\end{aligned}$ & $\begin{array}{l}F=1.24 \\
P=\end{array}$ & $\begin{array}{l}\mathrm{F}=0.99 \\
\mathrm{P}=0.5134\end{array}$ & $\begin{array}{l}F=4.06 \\
P=\end{array}$ & $\begin{array}{l}F=1.81 \\
P=\end{array}$ & $\begin{array}{l}F=1.47 \\
P=\end{array}$ & $\begin{array}{lr}\mathrm{F}= & 1.06 \\
\mathrm{P}=0.4258\end{array}$ & $\begin{array}{l}6 \mathrm{~F}=4.00 \\
3 \mathrm{P}=\end{array}$ & $\begin{array}{l}F=1.90 \\
P=0.0997\end{array}$ & $\begin{array}{l}\mathrm{F}= \\
1.34\end{array}$ \\
\hline $\begin{array}{l}\text { of } \\
\text { constants }\end{array}$ & $>5 \%$ & $<5 \%$ & $>5 \%$ & $>5 \%$ & $>5 \%$ & $>5 \%$ & $>5 \%$ & $\begin{array}{l}0.4214 \\
>5 \%\end{array}$ & $>5 \%$ & $\begin{array}{l}0.1379 \\
>5 \%\end{array}$ & $\begin{array}{l}0.1170 \\
>5 \%\end{array}$ & $\begin{array}{l}0.3320 \\
>5 \%\end{array}$ & $>5 \%$ & $\begin{array}{l}0.1405 \\
>5 \%\end{array}$ & $>5 \%$ & $\begin{array}{l}\mathrm{P}= \\
0.3788 \\
>5 \%\end{array}$ \\
\hline $\begin{array}{l}\text { Hausman } \\
\text { test }\end{array}$ & & $\begin{array}{l}\chi_{2}=25.34 \\
P=0.0003 \\
<0.05\end{array}$ & & & & & & & & & & & & & & \\
\hline $\begin{array}{l}\text { Estimation } \\
\text { method } \\
\text { retained }\end{array}$ & OLS & $\mathrm{FE}$ & OLS & OLS & OLS & OLS & OLS & OLS & OLS & OLS & OLS & OLS & OLS & OLS & OLS & OLS \\
\hline $\mathrm{R}^{2}$ & $\mathrm{R}^{2}=0.994$ & $8 R^{2}=0.0051$ & $1 \mathrm{R}^{2}=0.9$ & $4 R^{2}=0.4005$ & $5 R^{2}=0.9948$ & $8 R^{2}=0.525$ & $7 R^{2}=0.9973$ & $3 \mathrm{R}^{2} 0.42$ & $5 R^{2}=0.9949$ & & $3 \mathrm{R}^{2}=0.9975$ & $5 R^{2}=0$ & $2 \mathrm{R}^{2}=0.9948$ & $8 R^{2}=0.56$ & $6 \mathrm{R}^{2}=0$. & $3 R^{2}=0.38$ \\
\hline observations & $\mathrm{sn}=99$ & $\mathrm{n}=21$ & $\mathrm{n}=47$ & $\mathrm{n}=31$ & $n=99$ & $n=21$ & $n=47$ & $\begin{array}{l}0.0499 \\
n=31\end{array}$ & $\mathrm{n}=99$ & $\begin{array}{l}0.0819 \\
n=21\end{array}$ & $\begin{array}{l}\mathrm{P}=0.0000 \\
\mathrm{n}=47\end{array}$ & $\begin{array}{l}0.0708 \\
n=31\end{array}$ & $n=99$ & $\begin{array}{l}0.0793 \\
\mathrm{n}=21\end{array}$ & $n=47$ & $\begin{array}{l}0.0872 \\
\mathrm{n}=31\end{array}$ \\
\hline
\end{tabular}

The negative coefficient of earnings in some cases can be explained by the fact that, in an economic environment where there is frequency of losses (Collins and al, 1997 and Soussi and al, 2006), earnings can be considered as having been subject for earnings management and smoothing of earnings. Amir and Lev (1996) find a negative association between book value of equity and market value and a positive association between earnings and market value (in the model of market value of the firm as dependant variable and earnings and book value of equity as independent variables). Thus, when earnings and book value of equity are relevant in the same model, they have opposite signs. The negative association between the size of the firm and market value can be explained by the political costs. The addition of variables of disclosure to the accounting model makes the accounting numbers relevant to investors for low-tech firms. Thus, voluntary disclosure for these firms explains the irrelevance of accounting numbers. The significance of the change in $\mathrm{R}^{2}$ between the models is tested using the test of addition of variables. We use Chow test to study the difference of value relevance levels between subsamples. The level of relevance of the accounting model and of accounting model with control variables and sub-indexes of disclosure is low for low-tech firms and high-tech firms. The level of relevance of these models is high for medium-tech firms (these firms have an average technological level). The introduction of variables representing voluntary disclosure decreases significantly the relevance of accounting model for low-tech firms. For the high technology firms, the introduction of disclosure's variables increases insignificantly the relevance of the accounting model. Voluntary disclosure explains to a certain level low relevance of accounting information for these firms. Furthermore, the relevance of accounting model is higher for low technology firms than for high technology firms (the high technology firms have higher level of disclosure). 
Table 3: Results of multiple regressions (suite)

\begin{tabular}{|c|c|c|c|c|c|c|c|c|c|c|c|c|c|c|c|c|}
\hline \multirow{2}{*}{$\begin{array}{c}\text { Model } \\
\text { group } \\
\text { variable } \\
\end{array}$} & \multicolumn{4}{|c|}{$\begin{array}{l}\text { Basic model with introduction of } \\
\text { control variables and D4 }\end{array}$} & \multicolumn{4}{|c|}{$\begin{array}{l}\text { Basic model with introduction of } \\
\text { control variables and D5 }\end{array}$} & \multicolumn{4}{|c|}{$\begin{array}{l}\text { Basic model with introduction of } \\
\text { control variables and D6 }\end{array}$} & \multicolumn{4}{|c|}{$\begin{array}{l}\text { Basic model with introduction of } \\
\text { control variables and D7 }\end{array}$} \\
\hline & $\begin{array}{l}\text { Total } \\
\text { sample }\end{array}$ & LT & MT & HT & $\begin{array}{l}\text { Total } \\
\text { sample } \\
\end{array}$ & LT & MT & HT & $\begin{array}{l}\text { Total } \\
\text { sample }\end{array}$ & LT & MT & HT & $\begin{array}{l}\text { Total } \\
\text { sample }\end{array}$ & LT & MT & HT \\
\hline $\mathrm{BV}_{\mathrm{i}, \mathrm{t}} / \mathrm{P}_{\mathrm{i}, \mathrm{t}-1}$ & $\begin{array}{l}0.828 \\
(42.71)^{* * *}\end{array}$ & $\begin{array}{l}-0.328 \\
*(-1.19)\end{array}$ & $\begin{array}{l}0.815 \\
(36.58)^{* * * *}\end{array}$ & $\begin{array}{l}-0.310 \\
(-0.47)\end{array}$ & $\begin{array}{l}0.821 \\
(42.96)^{* * *}\end{array}$ & $\begin{array}{ll}0.675 \\
*(2.23)^{* *}\end{array}$ & $\begin{array}{l}0.808 \\
(38.90)^{* * *}\end{array}$ & $\begin{array}{c}-0.469 \\
*(-0.72)\end{array}$ & $\begin{array}{l}0.826 \\
(43.11)^{* * *}\end{array}$ & $\begin{array}{l}0.622 \\
*(1.71)\end{array}$ & $\begin{array}{l}0.815 \\
(38.04)^{* * *}\end{array}$ & $\begin{array}{l}0.202 \\
*(0.33)\end{array}$ & $\begin{array}{l}0.826 \\
(43.09)^{* * *}\end{array}$ & $\begin{array}{l}-0.352 \\
*(-1.72)\end{array}$ & $\begin{array}{l}0.812 \\
(37.87)^{* * *}\end{array}$ & $\begin{array}{c}-0.012 \\
*(-0.02)\end{array}$ \\
\hline $\mathrm{E}_{\mathrm{i}, \mathrm{t}} / \mathrm{P}_{\mathrm{i}, \mathrm{t}-1}$ & $\begin{array}{l}-2.518 \\
(-9.99)^{* * * *}\end{array}$ & $\begin{array}{l}- \\
17.948 \\
-- \\
3.46)^{* *}\end{array}$ & $\begin{array}{l}-2.719 \\
8(-9.12)^{* * *} \\
\end{array}$ & $\begin{array}{l}7.687 \\
(2.92)^{* * *}\end{array}$ & $\begin{array}{l}-2.646 \\
(- \\
10.59)^{* * *}\end{array}$ & $\begin{array}{l}5.316 \\
(1.30)\end{array}$ & $\begin{array}{l}-2.853 \\
(- \\
10.36)^{* * *}\end{array}$ & $\begin{array}{l}6.177 \\
(2.83)^{* * *}\end{array}$ & $\begin{array}{l}-2.554 \\
*(- \\
10.34)^{* * *}\end{array}$ & $\begin{array}{l}4.059 \\
(0.68)\end{array}$ & $\begin{array}{l}-2.746 \\
(-9.85)^{* * * *}\end{array}$ & $\begin{array}{l}5.747 \\
*(2.66)^{* *}\end{array}$ & $\begin{array}{l}-2.554 \\
(- \\
10.34)^{* * *}\end{array}$ & $\begin{array}{l}-17.447 \\
(-4.57)^{* *}\end{array}$ & $\begin{array}{l}-2.765 \\
(-9.89)^{* * *}\end{array}$ & $\begin{array}{l}6.322 \\
*(2.86)^{* * *}\end{array}$ \\
\hline D4 & $\begin{array}{l}-0.487 \\
(-0.63)\end{array}$ & $\begin{array}{l}2.657 \\
(2.06)\end{array}$ & $\begin{array}{l}-0.323 \\
(-0.28)\end{array}$ & $\begin{array}{l}0.719 \\
(0.35)\end{array}$ & & & & & & & & & & & & \\
\hline D5 & & & & & $\begin{array}{l}1.269 \\
(1.65)\end{array}$ & $\begin{array}{l}0.979 \\
(0.34)\end{array}$ & $\begin{array}{l}2.876 \\
(1.86)^{*}\end{array}$ & $\begin{array}{l}1.203 \\
(0.63)\end{array}$ & & & & & & & & \\
\hline D6 & & & & & & & & & $\begin{array}{l}0.237 \\
(0.34)\end{array}$ & $\begin{array}{l}-1.025 \\
(-0.28)\end{array}$ & $\begin{array}{l}1.367 \\
(0.81)\end{array}$ & $\begin{array}{l}-1.899 \\
(-1.26)\end{array}$ & & & & \\
\hline D7 & & & & & & & & & & & & & $\begin{array}{l}-0.269 \\
(-0.25)\end{array}$ & $\begin{array}{l}8.605 \\
(3.20)^{* *}\end{array}$ & $\begin{array}{l}1.587 \\
(0.83)\end{array}$ & $\begin{array}{l}-1.032 \\
(-0.51)\end{array}$ \\
\hline $\log \mathrm{TA}$ & $\begin{array}{l}-0.310 \\
(-4.73)^{* * * *}\end{array}$ & $\begin{array}{l}0.057 \\
(0.08)\end{array}$ & $\begin{array}{l}-0.299 \\
(-2.44)^{* *}\end{array}$ & $\begin{array}{l}-0.429 \\
(-2.18)^{* *}\end{array}$ & $\begin{array}{l}-0.325 \\
*(-5.06)^{* * *}\end{array}$ & $\begin{array}{l}-0.837 \\
(-0.91)\end{array}$ & $\begin{array}{l}-0.294 \\
(-2.50)^{* *}\end{array}$ & $\begin{array}{l}-0.247 \\
(-1.41)\end{array}$ & $\begin{array}{l}-0.285 \\
(-3.78)^{* * *}\end{array}$ & $\begin{array}{l}-0.453 \\
*(-1.00)\end{array}$ & $\begin{array}{l}-0.255 \\
(-1.89)^{*}\end{array}$ & $\begin{array}{l}-0.261 \\
(-1.54)\end{array}$ & $\begin{array}{l}-0.299 \\
(-4.73)^{* *}\end{array}$ & $\begin{array}{c}0.047 \\
*(0.09)\end{array}$ & $\begin{array}{l}-0.353 \\
(-2.60)^{* *}\end{array}$ & $\begin{array}{l}-0.243 \\
(-1.38)\end{array}$ \\
\hline Lev & $\begin{array}{l}0.326 \\
(1.25)\end{array}$ & $\begin{array}{l}2.056 \\
(2.87)^{*}\end{array}$ & $\begin{array}{r}0.437 \\
*(1.31)\end{array}$ & $\begin{array}{l}-0.653 \\
(-0.43)\end{array}$ & $\begin{array}{l}0.195 \\
(0.74)\end{array}$ & $\begin{array}{l}0.133 \\
(0.14)\end{array}$ & $\begin{array}{l}0.302 \\
(0.95)\end{array}$ & $\begin{array}{l}-0.677 \\
(-0.48)\end{array}$ & $\begin{array}{l}0.287 \\
(1.08)\end{array}$ & $\begin{array}{l}-0.108 \\
(-0.14)\end{array}$ & $\begin{array}{l}0.228 \\
(0.57)\end{array}$ & $\begin{array}{l}-0.074 \\
(-0.07)\end{array}$ & $\begin{array}{l}0.320 \\
(1.20)\end{array}$ & $\begin{array}{l}1.741 \\
(3.79)^{* *}\end{array}$ & $\begin{array}{l}0.299 \\
(0.85)\end{array}$ & $\begin{array}{l}0.088 \\
(0.07)\end{array}$ \\
\hline FP & $\begin{array}{l}0.326 \\
(5.28)^{* * *}\end{array}$ & $\begin{array}{l}28.877 \\
(4.80) \\
* *\end{array}$ & $\begin{array}{l}7.320 \\
(4.58)^{* * *}\end{array}$ & $\begin{array}{l}-1.406 \\
(-2.26)^{* *}\end{array}$ & $\begin{array}{l}0.330 \\
*(5.41)^{* * *}\end{array}$ & $\begin{array}{l}-0.606 \\
(-0.24)\end{array}$ & $\begin{array}{l}0.326 \\
(4.87)^{* * *}\end{array}$ & $\begin{array}{c}-0.904 \\
(-1.78)^{*}\end{array}$ & $\begin{array}{l}0.324 \\
(5.22)^{* * *}\end{array}$ & $\begin{array}{l}1.077 \\
(0.18)\end{array}$ & $\begin{array}{l}0.313 \\
(4.48)^{* * *}\end{array}$ & $\begin{array}{l}-0.905 \\
(-1.83)^{*}\end{array}$ & $\begin{array}{l}0.326 \\
(5.26)^{* * * *}\end{array}$ & $\begin{array}{l}28.555 \\
(6.41)^{* * *}\end{array}$ & $\begin{array}{l}0.324 \\
(4.68)^{* * *}\end{array}$ & $\begin{array}{l}-0.965 \\
(-1.84)^{*}\end{array}$ \\
\hline GR & $\begin{array}{l}4.19 \mathrm{E}-09 \\
(2.22)^{* *}\end{array}$ & $\begin{array}{l}5.11 \mathrm{E}- \\
09 \\
(1.03)\end{array}$ & $\begin{array}{l}5.08 \mathrm{E}-09 \\
(2.10)^{* *}\end{array}$ & $\begin{array}{l}1.27 \mathrm{E}-07 \\
(0.81)\end{array}$ & $\begin{array}{c}\text { 74.69E-09 } \\
(2.49)^{* *}\end{array}$ & $\begin{array}{l}4.14 \mathrm{E}-09 \\
(0.76)\end{array}$ & $\begin{array}{c}95.15 \mathrm{E}-09 \\
(2.24)^{* *}\end{array}$ & $\begin{array}{l}9.13 \mathrm{E}-08 \\
(0.58)\end{array}$ & $\begin{array}{c}84.18 \mathrm{E}-09 \\
(2.21)^{* *}\end{array}$ & $\begin{array}{l}2.19 \mathrm{E}-09 \\
(0.39)\end{array}$ & $\begin{array}{l}5.14 \mathrm{E}-09 \\
(2.16)^{* *}\end{array}$ & $\begin{array}{l}1.01 \mathrm{E}-07 \\
(0.65)\end{array}$ & $\begin{array}{l}4.18 \mathrm{E}-09 \\
(2.21)^{* *}\end{array}$ & $\begin{array}{l}-6.23 \mathrm{E}-09 \\
(-2.24)\end{array}$ & $\begin{array}{l}5.73 \mathrm{E}-09 \\
(2.31)^{* *}\end{array}$ & $\begin{array}{l}7.54 \mathrm{E}- \\
08 \\
(0.48)\end{array}$ \\
\hline $\begin{array}{l}\text { Test of } \\
\text { homogeneity }\end{array}$ & $\begin{array}{l}\mathrm{F}=1.08 \\
\mathrm{P}=0.4110\end{array}$ & $\begin{array}{l}\mathrm{F}= \\
12.20\end{array}$ & $\begin{array}{l}F=2.03 \\
P=0.07871\end{array}$ & $\begin{aligned} \mathrm{F} & =4.98 \\
\mathrm{P} & =\end{aligned}$ & $\begin{array}{l}8 \mathrm{~F}=1.00 \\
\mathrm{P}=\end{array}$ & $\begin{array}{l}F=5.01 \\
P=\end{array}$ & $\begin{array}{l}F=1.96 \\
P=0.0889\end{array}$ & $\begin{array}{l}F=1.58 \\
P=\end{array}$ & $\begin{array}{l}F=1.05 \\
P=0.4454\end{array}$ & $\begin{aligned} F & =5.49 \\
P & =0.0940\end{aligned}$ & $\begin{array}{l}F=1.90 \\
P=0.0995\end{array}$ & $\begin{aligned} F & =1.26 \\
P & =0.4130\end{aligned}$ & $\begin{array}{l}F=1.05 \\
P=0.4480\end{array}$ & $\begin{aligned} F & =19.10 \\
D P & =0.0167\end{aligned}$ & $\begin{array}{l}\mathrm{F}=1.89 \\
\mathrm{P}=\end{array}$ & $\begin{array}{l}F=2.05 \\
P=\end{array}$ \\
\hline $\begin{array}{l}\text { of } \\
\text { constants }\end{array}$ & $>5 \%$ & $\begin{array}{l}\mathrm{P}= \\
0.0317 \\
<5 \%\end{array}$ & $7^{>5 \%}$ & $\begin{array}{l}0.0280 \\
<5 \%\end{array}$ & $\begin{array}{l}0.5058 \\
>5 \%\end{array}$ & $\begin{array}{l}0.1056 \\
>5 \%\end{array}$ & $>5 \%$ & $\begin{array}{l}0.2987 \\
>5 \%\end{array}$ & $>5 \%$ & $>5 \%$ & $>5 \%$ & $>5 \%$ & $>5 \%$ & $<5 \%$ & $\begin{array}{l}0.1012 \\
>5 \%\end{array}$ & $\begin{array}{l}0.1915 \\
>5 \%\end{array}$ \\
\hline $\begin{array}{l}\text { Hausman } \\
\text { Test }\end{array}$ & & $\begin{array}{l}\chi_{2}= \\
19.91 \\
P= \\
0.0029 \\
<0.05\end{array}$ & & $\begin{array}{l}\chi_{2}= \\
3.46 \\
P= \\
0.7494 \\
>0.05\end{array}$ & & & & & & & & & & $\begin{array}{l}\chi_{2}=53.15 \\
P=0.0000 \\
<0.05\end{array}$ & & \\
\hline $\begin{array}{l}\text { Estimation } \\
\text { method } \\
\text { retained }\end{array}$ & OLS & $\mathrm{FE}$ & OLS & $\mathrm{RE}$ & OLS & OLS & OLS & OLS & OLS & OLS & OLS & OLS & OLS & $\mathrm{FE}$ & OLS & OLS \\
\hline $\begin{array}{l}\mathrm{R}^{2} \\
\text { and number }\end{array}$ & $\begin{array}{l}\mathrm{R}^{2}=0.9948 \\
\mathrm{~F}=2482.42\end{array}$ & $\begin{array}{l}3 R^{2}= \\
20.0009\end{array}$ & $\begin{aligned} \mathrm{R}^{2} & =0.9973 . \\
9 \mathrm{~F} & =2063.87\end{aligned}$ & $\begin{array}{l}3 R^{2}= \\
7.3551\end{array}$ & $\begin{array}{l}\mathrm{R}^{2}=0.9949 \\
\mathrm{~F}=2545.85\end{array}$ & $\begin{array}{l}9 \mathrm{R}^{2}=0.462 \\
5 \mathrm{~F}=1.60\end{array}$ & $\begin{aligned} 2 \mathrm{R}^{2} & =0.9975 \\
\mathrm{~F} & =2242.53\end{aligned}$ & $\begin{array}{l}5 \mathrm{R}^{2} 0.3955 \\
3 \mathrm{~F}=2.15\end{array}$ & $\begin{aligned} 5 R^{2} & =0.9948 \\
F & =2474.81\end{aligned}$ & $\begin{array}{l}8 R^{2}=0.4610 \\
1 F=1.59\end{array}$ & $\begin{aligned} 0 \mathrm{R}^{2} & =0.9973 \\
\mathrm{~F} & =2094.16\end{aligned}$ & $\begin{array}{l}3 \mathrm{R}^{2}=0.4250 \\
6 \mathrm{~F}=2.43\end{array}$ & $\begin{aligned} \mathrm{OR}^{2} & =0.9944 \\
\mathrm{~F} & =2473.2\end{aligned}$ & $\begin{array}{l}8 \mathrm{R}^{2}=0.0133 \\
4 \mathrm{~F}=30.18\end{array}$ & $\begin{array}{l}3 R^{2}= \\
0.9973\end{array}$ & $\begin{array}{l}\mathrm{R}^{2}= \\
0.3920\end{array}$ \\
\hline & $\mathrm{P}=0.0000$ & $F=$ & $P=0.0000$ & Wald & $P=0.0000$ & & $P=0.0000$ & $\mathrm{P}=$ & $P=0.0000$ & $P=0.2236$ & $5 \mathrm{P}=0.0000$ & $P=0.0510$ & $P=0.0000$ & $P=0.0088$ & $8 \mathrm{~F}=$ & $\mathrm{F}=2.12$ \\
\hline observations I & $\mathrm{n}=99$ & $\begin{array}{l}16.36 \\
P= \\
0.0213 \\
n=21\end{array}$ & $\mathrm{n}=47$ & $\begin{array}{l}\text { chi2(7) } \\
=17.23 \\
P= \\
0.0160 \\
n=31\end{array}$ & $\mathrm{n}=99$ & $\begin{array}{l}0.2209 \\
n=21\end{array}$ & $\mathrm{n}=47$ & $\begin{array}{l}0.0785 \\
\mathrm{n}=31\end{array}$ & $\mathrm{n}=99$ & $\mathrm{n}=21$ & $\mathrm{n}=47$ & $\mathrm{n}=31$ & $\mathrm{n}=99$ & $\mathrm{n}=21$ & $\begin{array}{l}2096.36 \\
P= \\
0.0000 \\
n=47\end{array}$ & $\begin{array}{l}\mathrm{P}= \\
0.0825 \\
\mathrm{n}=31\end{array}$ \\
\hline
\end{tabular}

\section{B- Voluntary Disclosure On Intangibles And Low Relevance Of Accounting Information}

\section{Literature Review}

There is a complementary relationship between traditional accounting information and non-financial information representing intangible expenses for high-tech firms (Amir and Lev, 1996). Lev and Zarowin (1999) show that the decline of the relevance of accounting earnings is due to the importance of intangibles none capitalized. Our goal in this second part is to investigate whether the voluntary disclosure on intangibles explains the low relevance of accounting information. Goodwin (2002) shows that relevance of earnings is decreasing for firms that do not recognize intangible assets and there is little evidence of decline for firms which recognize intangible assets. In addition, after controlling losses, capitalizers did not have a change in the relevance of earnings and non capitalizers have a decrease of earnings' relevance. Goodwin and Ahmed (2006) show that the difference of earnings' relevance between "capitalizers" firms and "non-capitalizers" firms is more important in the latter part of the 1990s and that this difference is increasing. Furthermore, Lopes (2003) find that accounting numbers are more relevant for high-tech firms than for traditional firms in Brazil where there is capitalization of intangibles. Moreover, Ahmed and Falk (2006) demonstrate that managerial discretion in accounting practice, to capitalize or to expense R \& D, offers a higher relevance than accounting numbers that are the product of mandatory expensing of R \& D. Of same, Wang (2000) shows that firms choose the accounting method to apply for software R \& D that is more informative about their values. Unlike the pre-cited researches, Oswald (2008) shows that «Expensers » firms have little gain or nothing to gain in terms of relevance when they adjust their earnings and book values of equity to reflect the numbers as if these firms were "capitalizers". Similarly, Cheng, Hsieh and Yip (2007) show that the accounting choice has no significant 
effect on the relevance of accounting information. Amir and Lev (1996) conclude that the disclosure on intangibles decreases the relevance of accounting information. Also, Lee, Press and Choi (2005) attribute the low relevance of earnings for high-tech firms relative to low-tech firms mainly to noise (non financial information). By studying Brazilian market, Lopes (2003) show that accounting numbers are more relevant for high-tech firms than for traditional firms. Lopes (2003) advance among the arguments of his results that in the emerging market studied, information sources for high tech firms are less available than in developed markets. Furthermore, Zhao (2002) shows that the level of disclosure on research and development has a significant effect on the relevance of accounting information.

\section{Methodology}

According to our literature review, we test the following hypotheses:

Hypothesis 3: The recognized intangibles are more relevant than disclosed intangibles.

Hypothesis 4: The level of disclosure on intangibles has an effect on the relevance of the accounting model for evaluating companies.

Hypothesis 5: The intensity of intangible expenditures (intangibles recognized in the income statement) has an effect on the relevance of the accounting model for evaluating companies.

Hypothesis 6: The weight of intangible assets (intangibles recognized on the balance sheet) has an effect on the relevance of the accounting model for evaluating companies

Sample and Data Collection: We study the same sample of Canadian companies of the first part.

Specification of models: We study the basic accounting model used in the first part and we introduce variables representing the intangibles :

Variable: Intensity of intangible expenses : The ratio of intensity of intangible expenses that we use is calculated as: The intensity of intangible expenses $=$ [expenditures on research and development + training costs + the spending on advertising, promotion and marketing expenses + management and organizational expenditures + expenditures for production process + software expenses + royalties paid (fees for concessions, patents, licenses, trademarks, processes, franchises, copyrights and reproduction; other similar rights and assets) + the spending assigned to quality standards + start-up and pre-operating costs + Other types of intangible expenses] / sales. Lev and Sougiannis (1996) use the indicators of intangible expenses intensity : annual R \& D / annual sales and annual expenses of advertising / annual sales.

Variable: Intangible assets: Intangible assets are those recognized in the balance sheet. We use the variable weight of intangible assets (Cazavan-Jeny, 2004).

Variable: Level of disclosure on intangibles: We use the index of disclosure on intangibles used in Trabelsi and Labelle (2006) (Appendix 4).

Multiple regressions: We estimate models that are detailed in the following.

Model 1 : Basic Model

$\frac{P_{i, t}}{P_{i, t-1}}=\alpha_{0}+\alpha_{1} \frac{E_{i, t}}{P_{i, t-1}}+\alpha_{2} \frac{B V_{i, t}}{P_{i, t-1}}+\varepsilon_{i, t}(1)$

$\varepsilon_{\mathrm{i}, \mathrm{t}}=$ residue.

i: icompany.

$\mathrm{t}$ : year ( $\mathrm{t}=1$ for $2006, \mathrm{t}=2$ for 2007).

$\mathrm{P}=$ Share price of the company three months after the end of the fiscal year.

$\mathrm{BV}=$ book value of equity of the company at the end of the fiscal year divided by the number of shares.

$\mathrm{E}=$ net income per share before extraordinary items of the company

Model 2: Basic model with the introduction of control variables and intensity of intangible expenses (intangibles recognized in the income statement)

$\underline{P_{i, t}}=a_{0}+a_{1} E_{i, t}+a_{2} \underline{B V_{i, t}}+a_{3} I_{i, t}+a_{4} \log T A_{i, t}+a_{5} G_{i, t}+a_{6} F_{i, t}+a_{7} L e V_{i, t}+\varepsilon_{i, t}$.

$\overline{\mathbf{P}_{\mathrm{i}, \mathrm{t}-1}} \overline{\mathbf{P}_{\mathrm{i}, \mathrm{t}-1}} \frac{\mathbf{P}_{\mathrm{i}, \mathrm{t}-1}}{\mathrm{P}}$

II = intensity of intangible expenditures $=$ intangible expenditures $/$ sales.

$\log \mathrm{TA}=\log$ of total assets ( $\log$ base 10$)$.

$\mathrm{GR}=$ growth rate $=$ change in sales from $\mathrm{t}$ to $\mathrm{t}-1$. 
FP = financial profitability or return = net income $/$ equity.

Lev $=$ Leverage $=$ long-term debt $/$ equity .

Model 3: Basic model with the introduction of control variables and the weight of intangible assets (intangibles recognized on the balance sheet)

$\underline{P_{i, t}}=b_{0}+b_{1} E_{i, t}+b_{2} \frac{B V_{i, t}}{P}+b_{3} I A_{i, t}+b_{4} \operatorname{LogTA}_{i, t}+b_{5} G_{i, t}+b_{6} F_{i, t}+b_{7} \operatorname{Lev}_{i, t}+\varepsilon_{i, t}$.

$\overline{\mathbf{P}_{\mathrm{i}, \mathrm{t}-1}} \quad \overline{\mathbf{P}_{\mathrm{i}, \mathrm{t}-1}} \overline{\mathbf{P}_{\mathrm{i}, \mathrm{t}-1}}$

$I A=$ intangible assets $=$ weight of intangible assets=intangible assets $/$ total assets.

Model 4: Basic model with the introduction of control variables and the index of disclosure on intangibles

$P_{i, t}=b_{0}+b_{1} E_{i, t}+b_{2} B V_{i, t}+b_{3} D 6_{i, t}+b_{4} \operatorname{LogTA}_{i, t}+b_{5} G_{i, t}+b_{6} F_{i, t}+b_{7} L_{e v} v_{i, t}+\varepsilon_{i, t}$.

$\mathbf{P}_{\mathrm{i}, \mathrm{t}-\mathrm{1}} \quad \mathbf{P}_{\mathrm{i}, \mathrm{t}-\mathrm{1}} \quad \mathbf{P}_{\mathrm{i}, \mathrm{t}-\mathrm{t}}$

D $6_{i, t}=$ index of disclosure on intangibles.

\section{Results and discussions of the second part}

Table 4: Results of multiple regressions

\begin{tabular}{|c|c|c|c|c|}
\hline Model & $\begin{array}{l}\text { Basic mode } \\
\text { and D6 }\end{array}$ & vith the intrc & oduction of & trol variables \\
\hline $\begin{array}{l}\text { Group } \\
\text { variable }\end{array}$ & $\begin{array}{l}\text { Total } \\
\text { sample }\end{array}$ & $\begin{array}{l}\text { Low } \\
\text { technology }\end{array}$ & $\begin{array}{l}\text { Medium } \\
\text { technology }\end{array}$ & $\begin{array}{l}\text { High } \\
\text { technology }\end{array}$ \\
\hline$B V_{i, t} / P_{i, t-1}$ & $\begin{array}{l}0.826 \\
(43.11)^{* * *}\end{array}$ & $\begin{array}{l}0.622 \\
(1.71)\end{array}$ & $\begin{array}{l}0.815 \\
(38.04)^{* * *}\end{array}$ & $\begin{array}{l}0.202 \\
(0.33)\end{array}$ \\
\hline $\mathrm{E}_{\mathrm{i}, \mathrm{t}} / \mathrm{P}_{\mathrm{i}, \mathrm{t}-\mathrm{1}}$ & $\begin{array}{l}-2.554 \\
(-10.34)^{* * *}\end{array}$ & $\begin{array}{l}4.059 \\
(0.68)\end{array}$ & $\begin{array}{l}-2.746 \\
(-9.85)^{* * *}\end{array}$ & $\begin{array}{l}5.747 \\
(2.66)^{* *}\end{array}$ \\
\hline D6 & $\begin{array}{l}0.237 \\
(0.34)\end{array}$ & $\begin{array}{l}-1.025 \\
(-0.28)\end{array}$ & $\begin{array}{l}1.367 \\
(0.81)\end{array}$ & $\begin{array}{l}-1.899 \\
(-1.26)\end{array}$ \\
\hline $\log T A$ & $\begin{array}{l}-0.285 \\
(-3.78)^{* * *}\end{array}$ & $\begin{array}{l}-0.453 \\
(-1.00)\end{array}$ & $\begin{array}{l}-0.255 \\
(-1.89)^{*}\end{array}$ & $\begin{array}{l}-0.261 \\
(-1.54)\end{array}$ \\
\hline Lev & $\begin{array}{l}0.287 \\
(1.08)\end{array}$ & $\begin{array}{l}-0.108 \\
(-0.14)\end{array}$ & $\begin{array}{c}0.228 \\
(0.57)\end{array}$ & $\begin{array}{l}-0.074 \\
(-0.07)\end{array}$ \\
\hline FP & $\begin{array}{l}0.324 \\
(5.22)^{* * *}\end{array}$ & $\begin{array}{l}1.077 \\
(0.18)\end{array}$ & $\begin{array}{l}0.313 \\
(4.48)^{* * *}\end{array}$ & $\begin{array}{l}-0.905 \\
(-1.83)^{*}\end{array}$ \\
\hline GR & $\begin{array}{l}4.18 \mathrm{E}-09 \\
(2.21)^{* *}\end{array}$ & $\begin{array}{l}2.19 \mathrm{E}-09 \\
(0.39)\end{array}$ & $\begin{array}{l}5.14 \mathrm{E}-09 \\
(2.16)^{* *}\end{array}$ & $\begin{array}{l}1.01 \mathrm{E}-07 \\
(0.65)\end{array}$ \\
\hline $\begin{array}{l}\mathrm{R}^{2} \\
\text { and number of observations }\end{array}$ & $\begin{array}{l}\mathrm{R}^{2}=0.9948 \\
\mathrm{~F}=2474.81 \\
\mathrm{P}=0.0000 \\
\mathrm{n}=99\end{array}$ & $\begin{array}{l}\mathrm{R}^{2}=0.4610 \\
\mathrm{~F}=1.59 \\
\mathrm{P}=0.2236 \\
\mathrm{n}=21\end{array}$ & $\begin{array}{l}\mathrm{R}^{2}=0.9973 \\
\mathrm{~F}=2094.16 \\
\mathrm{P}=0.0000 \\
\mathrm{n}=47\end{array}$ & $\begin{array}{l}\mathrm{R}^{2}=0.4250 \\
\mathrm{~F}=2.43 \\
\mathrm{P}=0.0510 \\
\mathrm{n}=31\end{array}$ \\
\hline
\end{tabular}

Legend of tables 4 and 5 :

FT: low-technology sectors, MT: medium-technology sectors, HT: high-tech sectors.

$\mathrm{Pi}, \mathrm{t}=$ Share price of the company three months after the end of the fiscal year.

$\mathrm{BV}=$ book value of equity of the company at the end of the fiscal year divided by the number of shares.

$\mathrm{E}=$ net income before extraordinary items per share of the company.

IA = intangible assets $=$ weight of intangible assets $=$ intangible assets $/$ total assets.

II = intensity of intangible expenditures= intangible expenditures / sales.

D6= index of disclosure on intangibles

$\log \mathrm{TA}=\log$ of total assets ( $\log$ base 10$)$.

$\mathrm{GR}=$ growth rate $=$ change in sales from $\mathrm{t}$ to $\mathrm{t}-1$.

$\mathrm{FP}=$ financial profitability or return $=$ net income $/$ equity.

Lev $=$ Leverage $=$ long-term debt $/$ equity .

$\mathrm{F}=$ value of the Fisher statistic.

P: probability of $F($ or $\chi 2$ ) greater than the calculated critical value of $F(\chi 2)$ or significance of $F($ or $\chi 2$ ).

OLS: ordinary least squares.

$\mathrm{R}^{2}$ : model's explanatory power.

$\mathrm{n}$ : number of observations. 
Values in parentheses are the values of t-statistics., * Significant at $10 \%,{ }^{* *}$ significant at $5 \%$, *** significant at $1 \%$

D6: Index of disclosure on intangibles.

Table 5: Summary of multiple regressions (suite)

\begin{tabular}{|c|c|c|c|c|c|c|c|c|c|c|c|c|c|c|c|c|}
\hline \multirow{2}{*}{$\begin{array}{l}\text { Model } \\
\text { Group } \\
\text { variable }\end{array}$} & \multicolumn{3}{|c|}{ Basic model } & \multirow[b]{2}{*}{ HT } & \multicolumn{4}{|c|}{$\begin{array}{l}\text { Basic model with introduction of control } \\
\text { variables }\end{array}$} & \multicolumn{4}{|c|}{$\begin{array}{l}\text { Basic model with introduction of } \\
\text { control variables and intangible } \\
\text { expenses intensity }\end{array}$} & \multicolumn{4}{|c|}{$\begin{array}{l}\text { Basic model with introduction of } \\
\text { control variables and weight of } \\
\text { intangible assets }\end{array}$} \\
\hline & $\begin{array}{l}\text { Total } \\
\text { sample }\end{array}$ & LT & MT & & $\begin{array}{l}\text { Total } \\
\text { sample }\end{array}$ & LT & MT & HT & $\begin{array}{l}\text { Total } \\
\text { sample }\end{array}$ & LT & MT & HT & $\begin{array}{l}\text { Total } \\
\text { sample }\end{array}$ & LT & MT & HT \\
\hline $\mathrm{BV}_{\mathrm{i}, \mathrm{t}} / \mathrm{P}_{\mathrm{i}, \mathrm{t}-1}$ & $\begin{array}{l}0.883 \\
(45.38)^{* * *}\end{array}$ & $\begin{array}{l}0.498 \\
(1.72)\end{array}$ & $\begin{array}{l}0.873 \\
(39.37)^{* * *}\end{array}$ & $\begin{array}{l}-0.205 \\
(-0.39)\end{array}$ & $\begin{array}{l}0.826 \\
(43.32)^{* * *}\end{array}$ & & $\begin{array}{l}0.814 \\
(38.35)^{* * *}\end{array}$ & $\begin{array}{l}-0.229 \\
(-0.44)\end{array}$ & $\begin{array}{l}0.947 \\
(8.18)^{* * *}\end{array}$ & $\begin{array}{l}1.182 \\
(1.29)\end{array}$ & $\begin{array}{l}0.668 \\
(5.54)^{* * *}\end{array}$ & $\begin{array}{l}-0.411 \\
(-0.75)\end{array}$ & $\begin{array}{l}0.828 \\
(44.00)^{* * *}\end{array}$ & $\begin{array}{l}0.098 \\
(0.22)\end{array}$ & $\begin{array}{l}0.815 \\
(39.61)^{* * *}\end{array}$ & $\begin{array}{l}-0.227 \\
(-0.43)\end{array}$ \\
\hline $\mathrm{E}_{\mathrm{i}, \mathrm{t}} / \mathrm{P}_{\mathrm{i}, \mathrm{t}-\mathrm{1}}$ & $\begin{array}{l}-1.725 \\
(-7.09)^{* * *}\end{array}$ & $\begin{array}{l}1.549 \\
(0.99)\end{array}$ & $\begin{array}{l}-1.904 \\
(-6.78)^{* * *}\end{array}$ & $\begin{array}{l}3.201 \\
(3.14)^{* * *}\end{array}$ & $\begin{array}{l}-2.551 \\
(- \\
10.39)^{* * *}\end{array}$ & & $\begin{array}{l}-2.747 \\
(-9.90)^{* * *}\end{array}$ & $\begin{array}{l}6.191 \\
(2.87)^{* * *}\end{array}$ & $\begin{array}{l}-0.857 \\
(-0.54)\end{array}$ & $\begin{array}{l}-2.731 \\
(-0.23)\end{array}$ & $\begin{array}{l}-4.773 \\
(- \\
2.89)^{* * *}\end{array}$ & $\begin{array}{l}10.071 \\
(2.68)^{* *}\end{array}$ & $\begin{array}{l}-2.519 \\
(- \\
10.38)^{* * *}\end{array}$ & $\begin{array}{l}6.589 \\
(1.75)\end{array}$ & $\begin{array}{l}-2.703 \\
(- \\
10.01)^{* * *}\end{array}$ & $\begin{array}{l}6.991 \\
(2.94) \\
* * *\end{array}$ \\
\hline II & & & & & & & & & $\begin{array}{l}0.699 \\
(2.11)^{* *}\end{array}$ & $\begin{array}{l}25.846 \\
(0.87)\end{array}$ & $\begin{array}{l}2.822 \\
(0.81)\end{array}$ & $\begin{array}{l}-0.252 \\
(-1.26)\end{array}$ & & & & \\
\hline IA & & & & & & & & & & & & & $\begin{array}{l}-0.741 \\
(-1.94)^{*}\end{array}$ & $\begin{array}{l}1.705 \\
(1.72)\end{array}$ & $\begin{array}{l}-1.333 \\
(-1.90)^{*}\end{array}$ & $\begin{array}{l}1.040 \\
(0.82)\end{array}$ \\
\hline $\log \mathrm{TA}$ & & & & & $\begin{array}{l}-0.299 \\
(-4.76)^{* * *}\end{array}$ & & $\begin{array}{l}-0.303 \\
(-2.50)^{* *}\end{array}$ & $\begin{array}{l}-0.224 \\
(-1.32)\end{array}$ & $\begin{array}{l}-1.403 \\
(-4.43 \\
)^{* * *}\end{array}$ & $\begin{array}{l}0.0775 \\
(0.09)\end{array}$ & $\begin{array}{l}-0.389 \\
(-2.18)^{* *}\end{array}$ & $\begin{array}{l}-0.276 \\
(-1.58)\end{array}$ & $\begin{array}{l}-0.304 \\
(-4.91)^{* * *}\end{array}$ & $\begin{array}{l}-0.157 \\
(-0.42\end{array}$ & $\begin{array}{l}-0.345 \\
(-2.89)^{* * *}\end{array}$ & $\begin{array}{l}-0.183 \\
(-1.03\end{array}$ \\
\hline Lev & & & & & $\begin{array}{l}0.306 \\
(1.18)\end{array}$ & & $\begin{array}{l}0.417 \\
(1.30)\end{array}$ & $\begin{array}{l}-0.179 \\
(-0.16)\end{array}$ & $\begin{array}{l}1.683 \\
(1.37)\end{array}$ & $\begin{array}{l}0.312 \\
(0.33)\end{array}$ & $\begin{array}{l}0.135 \\
(0.44)\end{array}$ & $\begin{array}{l}-0.428 \\
(-0.36)\end{array}$ & $\begin{array}{l}0.289 \\
(1.13)\end{array}$ & $\begin{array}{l}0.248 \\
(0.36)\end{array}$ & $\begin{array}{l}0.564 \\
(1.76)^{*}\end{array}$ & $\begin{array}{l}0.343 \\
(0.26)\end{array}$ \\
\hline FP & & & & & $\begin{array}{l}0.325 \\
(5.29)^{* * *}\end{array}$ & & $\begin{array}{l}0.321 \\
(4.66)^{* * *}\end{array}$ & $\begin{array}{l}-0.902 \\
(-1.80)^{*}\end{array}$ & $\begin{array}{l}2.768 \\
(1.98)^{*}\end{array}$ & $\begin{array}{l}6.879 \\
(0.69)\end{array}$ & $\begin{array}{l}2.108 \\
(1.69)\end{array}$ & $\begin{array}{l}-2.662 \\
(-1.77 \\
)^{*}\end{array}$ & $\begin{array}{l}0.334 \\
(5.49)^{* * *}\end{array}$ & $\begin{array}{l}0.926 \\
(0.38)\end{array}$ & $\begin{array}{l}0.335 \\
(4.99)^{* * *}\end{array}$ & $\begin{array}{l}-1.014 \\
(-1.94 \\
)^{*}\end{array}$ \\
\hline GR & & & & & $\begin{array}{l}4.17 \mathrm{E}-09 \\
(2.22)^{* *}\end{array}$ & & $\begin{array}{l}5.16 \mathrm{E}-09 \\
(2.17)^{* *}\end{array}$ & $\begin{array}{l}7.51 \mathrm{E}-08 \\
(0.49)\end{array}$ & $\begin{array}{l}1.14 \mathrm{E}-09 \\
(0.47)\end{array}$ & $\begin{array}{l}4.56 \mathrm{E}- \\
09 \\
(0.86)\end{array}$ & $\begin{array}{l}3.12 \mathrm{E}-09 \\
(1.32)\end{array}$ & $\begin{array}{l}-1.14 \mathrm{E}- \\
08 \\
(-0.07)\end{array}$ & $\begin{array}{l}3.54 \mathrm{E}-09 \\
(1.88)^{*}\end{array}$ & $\begin{array}{l}3.59 \mathrm{E}- \\
09 \\
(0.87)\end{array}$ & $\begin{array}{l}4.54 \mathrm{E}-09 \\
(1.95)^{*}\end{array}$ & $\begin{array}{l}1.84 \mathrm{E}- \\
07 \\
(0.90)\end{array}$ \\
\hline Test of & $\mathrm{F}=1.45$ & $\mathrm{~F}=$ & $\mathrm{F}=1.44$ & $\mathrm{~F}=0.95$ & $\mathrm{~F}=\quad 1.07$ & $\mathrm{~F}=$ & $\mathrm{F}=\quad 2.06$ & $F=1.57$ & $\mathrm{~F}=1.84$ & $\mathrm{~F}=$ & $\mathrm{F}=2.13$ & $\mathrm{~F}=1.19$ & $\mathrm{~F}=\quad 0.98$ & $\mathrm{~F}=$ & $F=2.07$ & $\mathrm{~F}=$ \\
\hline homo- & $\begin{array}{l}\mathrm{P}=0.1053 \\
>5 \%\end{array}$ & 1.42 & $\mathrm{P}=0.2047$ & $P=$ & $\begin{array}{l}\mathrm{P}= \\
0.4147\end{array}$ & $\begin{array}{l}6.50 \\
P=\end{array}$ & $\mathrm{P}=0.0693$ & $\mathrm{P}=$ & $\begin{array}{l}\mathrm{P}= \\
0.0399\end{array}$ & $\begin{array}{l}40.62 \\
P=\end{array}$ & $\begin{array}{l}\mathrm{P}= \\
0.1111\end{array}$ & $\mathrm{P}=$ & $\mathrm{P}=0.5299$ & $\begin{array}{l}6.15 \\
P=\end{array}$ & $\begin{array}{l}P= \\
0.0731\end{array}$ & $\begin{array}{l}2.18 \\
P=\end{array}$ \\
\hline $\begin{array}{l}\text { geneity } \\
\text { of } \\
\text { cons-tants }\end{array}$ & $>5 \%$ & $\begin{array}{l}P= \\
0.315> \\
5 \%\end{array}$ & $>5 \%$ & $\begin{array}{l}0.5514 \\
>5 \%\end{array}$ & $\begin{array}{l}0.4147 \\
>5 \%\end{array}$ & $\begin{array}{l}\mathrm{P}= \\
0.043 \\
<5 \%\end{array}$ & $>5 \%$ & $\begin{array}{l}0.2791 \\
>5 \%\end{array}$ & $\begin{array}{l}0.0399 \\
<5 \%\end{array}$ & $\begin{array}{l}P= \\
0.120> \\
5 \%\end{array}$ & $\begin{array}{l}0.1111 \\
>5 \%\end{array}$ & $\begin{array}{l}0.461> \\
5 \%\end{array}$ & $>5 \%$ & $\begin{array}{l}P= \\
0.080> \\
5 \%\end{array}$ & $\begin{array}{l}0.0731 \\
>5 \%\end{array}$ & $\begin{array}{l}\mathrm{P}= \\
0.170 \\
>5 \%\end{array}$ \\
\hline $\begin{array}{l}\text { Hausm-an } \\
\text { test }\end{array}$ & & & & & & $\begin{array}{l}\text { CHI2= } \\
-38.98 \\
\text { CHI2<0 }\end{array}$ & & & $\begin{array}{l}\chi_{2}= \\
21.87 \\
P=0.0013 \\
<0.05\end{array}$ & & & & & & & \\
\hline $\begin{array}{l}\text { Estimation } \\
\text { method } \\
\text { retained }\end{array}$ & OLS & OLS & OLS & OLS & OLS & & OLS & OLS & $\mathrm{FE}$ & OLS & OLS & OLS & OLS & OLS & OLS & OLS \\
\hline $\begin{array}{l}\mathrm{R}^{2} \\
\text { and num- } \\
\text { ber of } \\
\text { observations }\end{array}$ & $\begin{array}{l}\mathrm{R}^{2}= \\
0.9920 \\
\mathrm{~F}= \\
5981.36 \\
\mathrm{P}=0.0000 \\
\mathrm{n}=99\end{array}$ & $\begin{array}{l}\mathrm{R}^{2}= \\
0.174 \\
\mathrm{~F}= \\
1.90 \\
\mathrm{P}= \\
0.178 \\
\mathrm{n}=21\end{array}$ & $\begin{array}{l}\mathrm{R}^{2}= \\
0.9954 \\
\mathrm{~F}= \\
4785.29 \\
\mathrm{P}=0.0000 \\
\mathrm{n}=47\end{array}$ & $\begin{array}{l}\mathrm{R}^{2}= \\
0.2609 \\
\mathrm{~F}=4.94 \\
\mathrm{P}= \\
0.0145 \\
\mathrm{n}=31\end{array}$ & $\begin{array}{l}\mathrm{R}^{2}= \\
0.9948 \\
\mathrm{~F}= \\
2915.21 \\
\mathrm{P}=0.0000 \\
\mathrm{n}=99\end{array}$ & & $\begin{array}{l}\mathrm{R}^{2}= \\
0.9973 \\
\mathrm{~F}= \\
2464.68 \\
\mathrm{P}=0.0000 \\
\mathrm{n}=47\end{array}$ & $\begin{array}{l}\mathrm{R}^{2}= \\
0.3852 \\
\mathrm{~F}= \\
2.51 \\
\mathrm{P}= \\
0.0502 \\
\mathrm{n}=31\end{array}$ & $\begin{array}{l}\mathrm{R}^{2}= \\
0.9711 \\
\mathrm{~F}= \\
2485.85 \\
\mathrm{P}= \\
0.0000 \\
\mathrm{n}=83\end{array}$ & $\begin{array}{l}\mathrm{R}^{2}= \\
0.510 \\
\mathrm{~F}= \\
1.34 \\
\mathrm{P}= \\
0.3332 \\
\mathrm{n}=17\end{array}$ & $\begin{array}{l}\mathrm{R}^{2}= \\
0.9986 \\
\mathrm{~F}=2948 \\
\mathrm{P}= \\
0.0000 \\
\mathrm{n}=36\end{array}$ & $\begin{array}{l}\mathrm{R}^{2}= \\
0.4297 \\
\mathrm{~F}=2.37 \\
\mathrm{P}= \\
0.0577 \\
\mathrm{n}=30\end{array}$ & $\begin{array}{l}\mathrm{R}^{2}= \\
0.9950 \\
\mathrm{~F}= \\
2573.96 \\
\mathrm{P}=0.0000 \\
\mathrm{n}=99\end{array}$ & $\begin{array}{l}\mathrm{R}^{2}= \\
0.5579 \\
\mathrm{~F}= \\
2.34 \\
\mathrm{P}= \\
0.0877 \\
\mathrm{n}=21\end{array}$ & $\begin{array}{l}\mathrm{R}^{2}= \\
0.9975 \\
\mathrm{~F}= \\
2250.52 \\
\mathrm{P} \\
0.0000 \\
\mathrm{n}=47\end{array}=$ & $\begin{array}{l}\mathrm{R}^{2}= \\
0.402 \\
\mathrm{~F}= \\
2.22 \\
\mathrm{P}= \\
0.0709 \\
\mathrm{n}=31\end{array}$ \\
\hline
\end{tabular}

We find a negative association and significant only at $10 \%$ between the weights of intangible assets and stock market prices (as in Ely and Waymire, 1999). This relation shows that the capitalization of intangible issues a negative signal to investors. The intangibles recognized are more relevant than intangibles disclosed only for the total sample and medium-tech firms. The level of relevance of the accounting model with control variables and recognized and disclosed intangibles is low for low technology firms and high technology firms. The level of relevance of these three models is high for medium-tech firms. For the model 3 in the table 6, there is no difference of $\mathrm{R}^{2}$ for the three subsamples. However, there is a difference between the $\mathrm{R}^{2}$ for the three sub-samples for the model 4 in table 5 and the model in table 5 . The $\mathrm{R}^{2}$ of low-tech firms is higher than the $\mathrm{R}^{2}$ of high technology firms for the three models above (high tech firms have higher level of relevance). The introduction of intangibles recognized and disclosed in the accounting model increases not significantly the relevance of this model with one exception for the total sample. The intensity of intangible expenditures has a significant effect on the relevance of the accounting model for the total sample.

\section{General Conclusion}

For the basic model, we find a low level of relevance for high technology firms and low technology firms. Medium-tech firms have a high level of relevance (due to average technological level). The introduction of the overall disclosure index and sub-indexes of disclosure decreases significantly the relevance of the accounting model for low- tech firms. For high technology firms, it increases insignificantly the relevance of the accounting model. Thus, voluntary disclosure explains to a certain level the low relevance of accounting information. The relevance of accounting model is higher for low tech firms than for high tech firms (high tech firms have higher level of disclosure). Furthermore, the addition of variables of disclosure to the accounting model makes the accounting numbers relevant to investors for low-tech 
firms. Thus, voluntary disclosure for these firms explains the irrelevance of accounting numbers. For medium-tech firms, book value and earnings are relevant. For high technology firms, the earnings are relevant but the book value of equity is not relevant due to the capitalization of intangibles. This capitalization could be used as a signal and as a means of earnings management. In the second part of our research, we show that the inclusion of intangibles recognized and disclosed increases none significantly the relevance of the accounting model. Medium-tech firms have a high level of relevance of the accounting model, while the level of relevance of low-tech firms and high - tech firms is low. Dontoh et al (2004) and Lim and al (2011) show that the increased noise in stock returns over time is the primary factor of the decrease in the relevance of earnings. We could consider that the application of IFRS could result in higher levels of relevance of accounting information. However, Narktabtee et al (2011) and Barth et al (2008) show that it is not the application of IFRS that provides higher quality of accounting information and that improving the quality of accounting information depends both on the characteristics of the firm and the country.

Recommendations: Other factors have an impact on the relevance of accounting information. For example, Cottle et al. (1998) argue that the increasing use of the balance sheet by investors is explained by the change in ownership structure. On the other hand, conservatism can be seen as another factor explaining the low relevance of accounting information. However, Balachandran and Mohanram, (2011) find that firms with a growing level of conservatism do not have more declining relevance of accounting information. Researches could study more these factors explaining low level of relevance.

\section{References}

Aboody, D. \& Lev, B. (1998). The value relevance of intangibles: The case of software capitalization. Journal of Accounting Research, Supplement 1998, 161-191.

Ahmed, K. \& Falk, H. (2006). The value relevance of management's research and development reporting choice: Evidence from Australia. Journal of Accounting and Public Policy, 25(3), 231.

Allen, S. (2003). Understanding book value for investors. Canadian stock picks and stock research, investorsfriends.com.

Al-Shammari, B. \& Al-Sultan, W. (2010).Corporate governance and voluntary disclosure in Kuwait. International Journal of Disclosure and Governance, 7(3), 262-280.

Amir, E. \& Lev, B. (1996). Value-relevance of non financial information: the wireless communications industry. Journal of Accounting and Economics, 22, 3-30.

Balachandran, S. \& Mohanram, P. (2011). Is the decline in the value relevance of accounting driven by increased conservatism? Review of Accounting Studies, 16(2), 272.

Banghøj, J. \&Plenborg, T. (2008). Value relevance of voluntary disclosure in the annual report. Accounting and Finance, 48(2), 159.

Barth, M. E., Beaver, W. H. \& Landsman, W. R. (1998). Relative valuation roles of equity book value and net income as a function of financial health. Journal of Accounting and Economics, 25, 1-34.

Barth, M. E., Landsman, W. R. \& Lang, M. H. (2008). International Accounting Standards and Accounting Quality. Journal of Accounting Research, 46(3), 467.

Beattie, V., Goodagre, A. \&Thomson, S. (2000). Recognition versus disclosure: An investigation of the impact on equity risk using UK operating lease disclosures. Journal of Business, Finance and Auditing, 27(9) and (10).

Bernard, V. \& Schipper, K. (1994). Recognition and disclosure in financial reporting. working paper, University of Michigan and University of Chicago.

Botosan, C. A. (1997). Disclosure level and the cost of equity capital. The Accounting Review, 72(3), 323350.

Brown, S., Lo, K. \& Lys, T. (1999). Use of $\mathrm{R}^{2}$ in accounting research: measuring changes in value relevance over the last four decades. working paper, Northwestern University.

Callimaci, A. \& Landry, S. (2004). Market valuation of Research and Development Spending under Canadian GAAP. Canadian Accounting Perspectives, 3(1), 33.

Cazavan-Jeny, A. (2004). Le ratio market-to-book et la reconnaissance des immatériels- une étude du marché français. Comptabilité-Contrôle-Audit, 10 (2), 99-124.

Chalmers, K., Clinch, G. \& Godfrey, J. M. (2011). Changes in value relevance of accounting information upon IFRS adoption: Evidence from Australia. Australian Journal of Management, 36(2), 151-173.

Chang, J. (1999). The decline in value relevance of earnings and book values. working paper, University of Pennsylvania, Philadelphia, January 1999. 
Cheng, C. S. A., Hsieh, S. J. \& Yip, Y. (2007). Impact of accounting choices on firm valuation and earnings Quality The case of transition obligation under SFAS 106. Review of Accounting and Finance, 6(4), 419-441.

Christie, A. (1987). On cross-sectional analysis in accounting research. Journal of Accounting and Economics, 9, 231-258.

Collins, D., Maydew, E. \& Weiss, I. (1997).Changes in the value-relevance of earnings and book values over the past forty years. Journal of Accounting and Economics, 24(1), 39-68.

Cottle, S., Murray, R. \& Block, F. (1998). Graham and Dodd's security analysis. 5th ed, New York: McGraHill.

Ding, Y., Entwistle, G. \& Stolowy, H. (2004). International differences in research and development reporting practices: a French and Canadian comparison. Advances in International Accounting, 17, 55-72.

Dontoh, A., Radhakrishnan, S. \& Ronen, J. (2004). The Declining Value-relevance of Accounting Information and Non-information-based Trading: An Empirical Analysis. Contemporary Accounting Research, 21(4), 795-18.

Ely, K. \& Waymire, G. (1999). Intangible assets and stock prices in the Pre-SEC Era. Journal of Accounting Research, 37, 17-51.

Gelb, D. S. \& Zarowin, P. (2002). Corporate Disclosure Policy and the Informativeness of Stock Prices. Review of Accounting Studies, 7, 33-52.

Goodwin, J. (2002). Longitudinal Earnings Value Relevance and Intangible Assets: Evidence from Australian Firms, 1975-99. March 2002, working paper, Trobe University, School of Business, www.ssrn.com.

Goodwin, J. \& Ahmed, K. (2006).Longitudinal value relevance of earnings and intangible assets: Evidence from Australian firms. Journal of International Accounting, Auditing and Taxation, 15, 72-91.

Healy, P., Hutton, A. \& Palepu, K. (1999a). Stock performance and intermediation changes surrounding sustained increases in disclosure. Contemporary Accounting Research, 16(2).

Healy, P., Myers, S. \& Howe, C. (1999b). R\&D accounting and the tradeoff between relevance and objectivity. working paper, Harvard Business School.

Holthausen, R. W. \& Watts, R. L. (2001). The relevance of the value-relevance literature for financial accounting standard setting. Journal of Accounting and Economics, 31, 3-75.

Lapointe-Antunes, P., Cormier, D., Magnan, M. \& Gay-Angers, S. (2006). On the Relationship between Voluntary Disclosure, Earnings Smoothing and the Value-Relevance of Earnings: The Case of Switzerland. European Accounting Review, 15(4), 465.

Lee, B. B., Press, E. \& Choi, B. B. (2005).Why is the Value Relevance of Earnings Lower for High-Tech Firms? January 2005, working paper, www.ssrn.com.

Lev, B. (1989). On the usefulness of earnings and earnings research: Lessons and directions from two decades of empirical research, Journal of Accounting Research, Supplement 1989, 153-192.

Lev, B. \& Sougiannis T. (1996). The capitalization, amortization and value relevance of Research and Development. Journal of Accounting and Economics, February 1996, 107-138.

Lev, B. \& Zarowin, P. (1999). The boundaries of financial reporting and how to extend them. Journal of Accounting Research, 37 (2), 353-385.

Lo, K. (2004). The effect of scale differences on inferences in accounting research coefficient estimates, tests of incremental association and relative value relevance. Working paper, September 2004.

Lopes, A. B. (2003).The Value Relevance of Brazilian Accounting Numbers: An Empirical Investigation. Working paper, University of Sao Paulo, May 2003.

Lang, M. \& Lundholm, R. (1993). Cross-sectional determinants of analysts' ratings of corporate disclosures. Journal of Accounting Research, 31, 246-271.

Lim, S. C. \& Park, T. (2011). The declining association between earnings and returns; Diminishing value relevance of earnings or noisier markets? Management Research Review, 34(8), 947.

Lundholm, R. \& Myers, L. A. (2002).Bringing the future forward: The effect of disclosure on the returns earnings relation. Journal of Accounting Research, 40, 809-839.

Luo, S., Courtenay, S. M. \& Hossain, M. (2006).The effect of voluntary disclosure, ownership structure and proprietary cost on the return-future earnings relation. Pacific-Basin Finance Journal, 14, 501521.

Narktabtee, K. \& Patpanichchot, S. (2011).The impact of country - level versus firm - level factors on the effectiveness of IFRS adoption: The case of European Union. International Business and Economics Research Journal, 10(10).

Ohlson, J. A. (1995). Earnings, book value, and dividends in equity valuation. Contemporary accounting research, 11, 661-687. 
Oswald, D. R. (2008). The Determinants and Value Relevance of the Choice of Accounting for Research and Development Expenditures in the United Kingdom. JOURNAL, 35(1/2), 1.

Ramesh, K. \& Thiagarajan, R. (1995). Inter-temporal Decline in Earnings Response Coefficients. Working Paper, Northwestern University, Evanston, IL.

Soussi, J. C., Matoussi, H. \& Mouelhi, R. (2006). Intangibles and low relevance of accounting information. Master thesis in accounting, ISCAE of Tunis, may 2006.

Trabelsi, S. \& Labelle, R. (2006). Evidence that Corporate Websites is a Part of the Firm's Overall Disclosure Package. May 2006, working paper, www.google.com.

Wang, W. (2000).Discretionary choices of accounting methods and value relevance: R\&D accounting in the software industry. Ph.D. Dissertation, Washington University, 57 pages, http://proquest.umi.com.

Zhao, R. (2002). Relative value relevance of research and development reporting: An international comparison. Journal of International Financial Management and Accounting, 13(2), 153-174.

\section{Appendix 1: Present the Disclosure index used in Trabelsi and Labelle (2006)}

Appendix 1: Disclosure Index Scoring Sheet

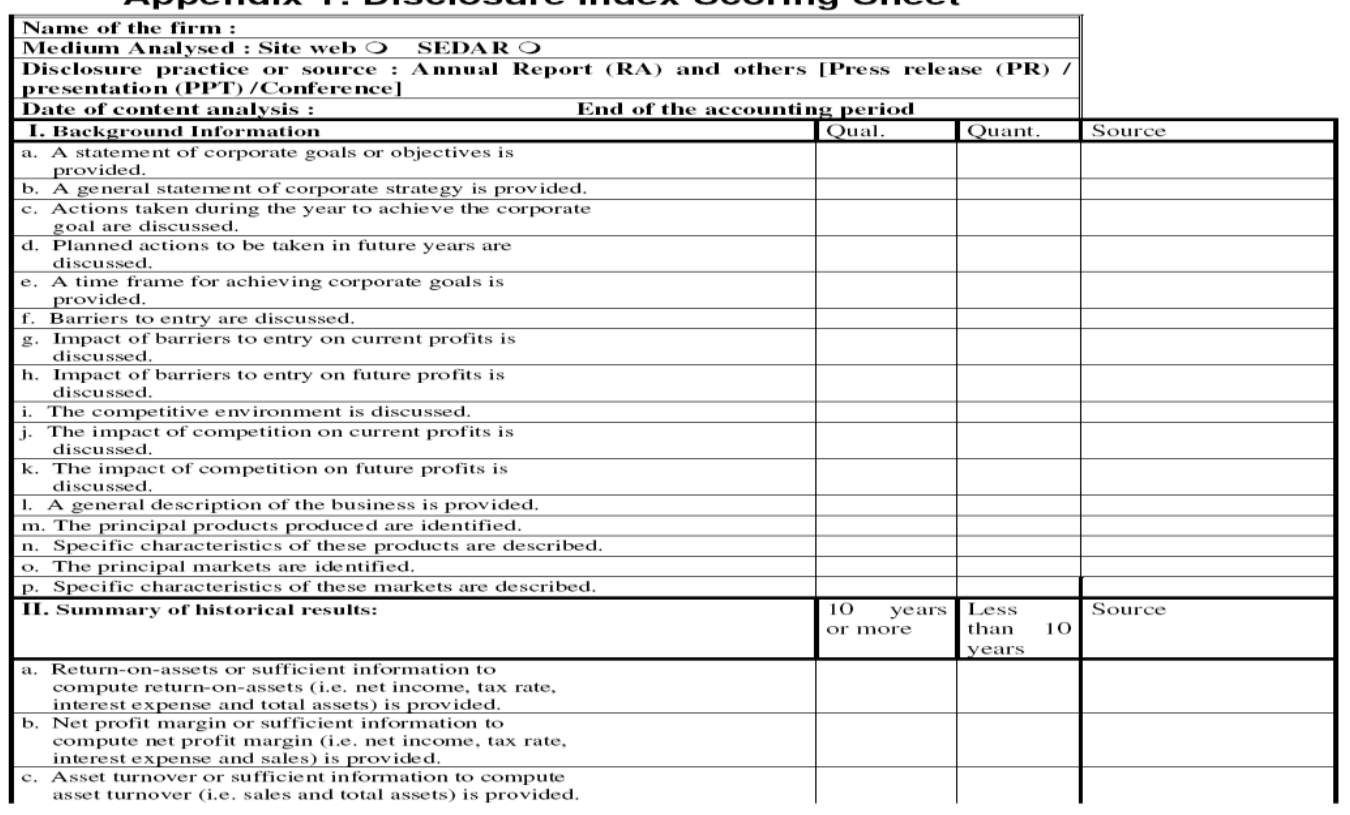

1. Our selection is based on items included in similar previous studies like the FASB report, 2001; the CICA report, 2001; Botosan, 1997; UQAM-CMA project "the best annual report" piloted by Coté and Gagnon-
Valotaire, 1992; Marston and Shrives, 1991; Merton, 1987; Healy and Palepu, 1993, 2001; Wallace, Naser and Mora, 1994; Cooke, 1989, 1992; Buzby, 1974, 1975; Firer and Meth, 1986; Choi, 1973; Dhaliwal.

\begin{tabular}{|c|c|c|c|}
\hline Social and environmental Information: & Qualitative & Quantitative & $\begin{array}{l}\text { Soure } \\
\text { e }\end{array}$ \\
\hline a. A statement of corporate social goal is provided & & & \\
\hline $\begin{array}{l}\text { b. Description of the engagement of the firm in the society by specific } \\
\text { social project }\end{array}$ & & & \\
\hline c. Description of the donation, grant, financial assistance & & & \\
\hline d. A statement of corporate environmental goal is provided & & & \\
\hline $\begin{array}{l}\text { e. Description of the activities to reduce the pollution related to the } \\
\text { activities of the firm }\end{array}$ & & & \\
\hline $\begin{array}{l}\text { f. Description of the actions taken for the transportation, the treatment } \\
\text { of the wastes }\end{array}$ & & & \\
\hline $\begin{array}{l}\text { g. Description of the activities of preservation et de restoration of the } \\
\text { green spaces and natural sites. }\end{array}$ & & & \\
\hline
\end{tabular}

\begin{tabular}{|c|c|c|c|}
\hline Social and environmental Information: & Qualitative & Quantitative & $\begin{array}{l}\text { Sourc } \\
\text { e }\end{array}$ \\
\hline a. A statement of corporate social goal is provided & & & \\
\hline $\begin{array}{l}\text { b. Description of the engagement of the firm in the society by specific } \\
\text { social project }\end{array}$ & & & \\
\hline c. Description of the donation, grant, financial assistance & & & \\
\hline d. A statement of corporate environmental goal is provided & & & \\
\hline $\begin{array}{l}\text { e. Description of the activities to reduce the pollution related to the } \\
\text { activities of the firm }\end{array}$ & & & \\
\hline $\begin{array}{l}\text { f. Description of the actions taken for the transportation, the treatment } \\
\text { of the wastes }\end{array}$ & & & \\
\hline $\begin{array}{l}\text { g. Description of the activities of preservation et de restoration of the } \\
\text { green spaces and natural sites. }\end{array}$ & & & \\
\hline
\end{tabular}




\begin{tabular}{|l|l|l|l|l|}
\hline & $\begin{array}{l}\text { Positive, } \\
\text { Negative } \\
\text { or } \\
\text { Neutral }\end{array}$ & $\begin{array}{l}\text { Qualitative } \\
\text { or } \\
\text { Quantitative }\end{array}$ & Source \\
\hline h. A projection of future profits is provided. & & & \\
\hline i. A projection of future sales is provided. & & & \\
\hline $\begin{array}{l}\text { Management discussion and analysis: } \\
\text { explanations for changes must be provided) }\end{array}$ & $\begin{array}{l}\text { Qualitative } \\
\text { or } \\
\text { Quantitative }\end{array}$ & $\begin{array}{l}\text { Segment of } \\
\text { production }\end{array}$ & $\begin{array}{l}\text { Total } \\
\text { Firm }\end{array}$ & Source \\
\hline a. Change in sales. & & & & \\
\hline b. Change in operating income. & & & \\
\hline & $\begin{array}{l}\text { Qualitative. } \\
\text { or } \\
\text { Quantitative }\end{array}$ & Total & Firm & Source \\
\hline c. Change in cost of goods sold. & & & & \\
\hline d. Change in cost of goods sold as a percentage of sales. & & & \\
\hline e. Change in gross profit. & & & \\
\hline f. Change in gross profit as a percentage of sales. & & & \\
\hline g. Change in selling and administrative expenses. & & & \\
\hline h. Change in interest expense or interest income. & & & \\
\hline i. Change in net income. & & & \\
\hline j. Change in inventory. & & & \\
\hline k. Change in accounts receivable. & & & \\
\hline l. Change in capital expenditures or R\&D. & & & \\
\hline m. Change in market share. & & & \\
\hline
\end{tabular}

Categories Added to the Botosan (1997) disclosure index

\begin{tabular}{|l|l|l|l|}
\hline Intangibles assets & Qualitative & Quantitative & Source \\
\hline a. Description of the major customers & & & \\
\hline b. Discussion of the investment in the customer service & & & \\
\hline c. Description of the size and the productivity of the sale force & & & \\
\hline d. Description des the major suppliers & & & \\
\hline e. Information on the employees affected to R\&D & & & \\
\hline f. Information on the R\&D program & & & \\
\hline g. Information on the R\&D alliances & & & \\
\hline h. Discussion of the importance of the trademark used & & & \\
\hline i. Discussion of the licences exploited & & \\
\hline
\end{tabular}


Appendix 2:Grouping of industries by level of knowledge (Canadian classification)

\begin{tabular}{|c|c|c|}
\hline High level & Medium level & Low level \\
\hline $\begin{array}{l}\text { Scientific and professional equipment } \\
\text { (M) }\end{array}$ & Other transport equipment (M) & Fishing and trapping $(\mathrm{P})$ \\
\hline $\begin{array}{l}\text { Communications equipment and } \\
\text { other electronic equipment (M) }\end{array}$ & $\begin{array}{l}\text { Other electrical and electronic } \\
\text { products (M) }\end{array}$ & $\begin{array}{l}\text { Other manufactured products } \\
\text { (M) }\end{array}$ \\
\hline aerospace (M) & Primary metals, nonferrous (M) & wood $(\mathrm{M})$ \\
\hline Computer and related services (S) & textiles $(\mathrm{M})$ & Furniture and furnishing (M) \\
\hline Office machines (M) & communications $(\mathrm{S})$ & Felling and forestry $(\mathrm{P})$ \\
\hline Engineering and scientific services (S) & Paper and allied products (M) & Transport (S) \\
\hline pharmaceuticals (M) & Mines $(\mathrm{P})$ & Storage and Warehousing (S) \\
\hline electrical energy (S) & rubber $(\mathrm{M})$ & Agriculture (P) \\
\hline Other chemicals (M) & Plastic (M) & Retail business (S) \\
\hline machinery (M) & Primary metals, ferrous (M) & personal services $(\mathrm{S})$ \\
\hline Refined petroleum and coal (M) & Non-metallic mineral (M) & Quarries and sand $(\mathrm{P})$ \\
\hline Management Consulting Services (S) & Wholesale trade $(\mathrm{S})$ & $\begin{array}{l}\text { Accommodation and } \\
\text { restoration }(S)\end{array}$ \\
\hline educational services (S) & Crude oil and natural gas (P) & clothing (M) \\
\hline Health and Social Services (S) & metal products (M) & leather $(\mathrm{M})$ \\
\hline Pipeline Transportation (S) & Automobiles and parts (M) & \\
\hline \multirow[t]{8}{*}{ Other business services (S) } & foodstuffs (M) & \\
\hline & drinks (M) & \\
\hline & tobacco $(\mathrm{M})$ & \\
\hline & Finance, insurance, real estate (S) & \\
\hline & Other utilities (S) & \\
\hline & Printing and publishing (M) & \\
\hline & Construction (S) & \\
\hline & Amusement and leisure(S) & \\
\hline
\end{tabular}

Note's: Primary, M: Manufacturing, S: Services

Source: Lee(1996) 1in Carroll(1998, p. 2) $2^{4}$

Appendix 3: Methods of classification of firms in high technology firms and traditional firms

\begin{tabular}{ll}
\hline Research & $\begin{array}{l}\text { Method for classifying firms into traditional firms and } \\
\text { HT firms }\end{array}$ \\
\hline Collins, Maydew and Weiss (1997) & Classification according to « two-digit SIC codes » \\
Barth, Beaver and Landsman (1998) & $\begin{array}{l}\text { Membership in high technology sectors: pharmaceutical } \\
\text { industry (classification according to the SIC codes) as a } \\
\text { proxy for high-tech sectors. } \\
\text { Classification depending on whether the sectors expected } \\
\text { to have significant unrecognized intangibles, to verify the } \\
\text { classification obtained, they compared the R \& D } \\
\text { expenditures as a percentage of total assets and ratio of } \\
\text { market-to-book of the two subsamples } \\
\text { Classification according to the ratio market-to-book: the } \\
\text { high-tech companies are those with the four highest } \\
\text { deciles of the ratio market-to-book and traditional } \\
\text { businesses have the lowest four deciles of the ratio. }\end{array}$ \\
\hline
\end{tabular}

\section{Appendix 4: Scoring Procedures (Trabelsi and Labelle, 2006) Background Information:}

Corporate goals: One point is awarded if the annual report includes a statement of the corporate goal or mission. One additional point is awarded if the goal is stated in quantitative terms, e.g.

ROE of $X \%$. Maximum points $=2.0$ 
Statement of business strategy: One point is awarded if the annual report includes a general statement of corporate strategy. One point is awarded if specific actions taken during the current year are outlined and one additional point is given if this includes quantitative information. One point is given if the annual report outlines specific actions to be taken in future years and one additional point is given if this includes quantitative information. One point is awarded if the annual report gives a time frame for attaining the corporate goal. Maximum points $=6.0$

Competition: One point is awarded if the annual report discusses barriers to entry. One point is awarded if the report discusses the impact of these on current firm profits and an additional point is given if the impact is quantified. One point is awarded if the annual report discusses the impact of barriers to entry on future firm profits and an additional point is given if this impact is quantified. One point is awarded if the report discusses the intensity of competition. One point is awarded if the impact of this on current firm profits is discussed and an additional point is given if this impact is quantified. One point is awarded if the report discusses the impact of competition on future firm profits and one additional point is given if this impact is quantified. Maximum points $=10.0$

Description of the business: One point is awarded if the annual report gives a general description of the companies' business activities. Maximum points $=1.0$

Principal products: One point is awarded if the annual report lists the principal products produced. One point is awarded if the characteristics of these products are described and an additional point is given if the description includes specific, quantified information. e.g. Our machine can produce widgets 5 times faster than the competitors' similar machines. Maximum points $=3.0$.

Principal markets: One point is awarded if the report lists the principal markets that buy the firm's products. One additional point is awarded if this is quantified. e.g. \% of sales to each market. One point is awarded if the annual report describes these markets and one additional point is given if this description is quantified. e.g. the size of the heavy duty truck market in 1989 was $X$ units. Maximum points $=4.0$

\section{Summary of historical results}

Annual summary: One point is awarded if sufficient information is given to compute return-on-assets for 9 or fewer years (typically 5 or 10 years is given). One additional point is awarded if sufficient information is given to compute return-on-assets for 10 or more years. One point is awarded if sufficient information is given to compute net profit margin for 9 or fewer years and one additional point is given if sufficient information is available to compute net profit margin for ten or more years. One point is awarded if sufficient information is given to compute asset turnover for 9 or fewer years. One additional point is awarded if sufficient information is available to compute asset turnover for 10 or more years. Since any two of ROA, profit margin or asset turnover is sufficient to compute the third, companies may only earn point for two of the three if all three are presented. One point is awarded if sufficient information is available to compute return-on-equity for 9 or fewer years and one additional point is awarded if sufficient information is available to compute return-on-equity for 10 or more years. Maximum points $=6.0$

Quarterly summary: One point is awarded if quarterly sales and net income is available for at least the most recent 8 quarters. Maximum points $=1.0$

Key non-financial statistics: Two points are awarded for each of the 20 items listed. In some instances firms may provide one item more than once. For example, several market share figures may be present if the firm operates in more than one line of business. Only the first instance of disclosure is counted. Maximum points $=40.0$

\section{Projected information}

Comparison of last year forecast to actual: One point is awarded if the annual report includes a comparison of a prior earnings projections to this years actual. One additional point is given if this comparison is quantified. One point is awarded if the annual report includes a comparison of prior sales 
projections to these years actual. One additional point is given if this comparison is quantified. Maximum points $=4.0$

Anticipated impact of opportunities and /or risks: Two points are awarded if the report discusses the profit or sales implications of opportunities available to the firm. One additional point is given if these implications are quantified. Two points are awarded if the report discusses the profit or sales implications of risks facing the firm. One additional point is given if these implications are quantified. Maximum points $=6.0$

Projected market share: Two points are awarded if the annual report includes a forecast of future market share and one additional point is given if this is quantified. Maximum points $=3.0$

Projected cash flow: Two points are given if the firm provides a discussion of future cash flow requirements and how they will be met. One additional point is given if these requirements are quantified. Maximum points $=3.0$

Projected capital and/or research and development expenditures: Two points are given if the annual report includes a discussion of future capital expenditures other than those already committed to. One additional point is given if the amount of planned capital expenditures is given. Two points are given if the annual report includes a discussion of future expenditures on research and development. One additional point is give in the amount of planned research and development expenditures is given. Maximum points $=6.0$

Earnings and/or sales projections: For a single segment firm, two points are given if the firm provides a profit projection and one additional point is given if the projection is a point estimate. Two points are given if the firm provides a sales projection and one additional point is given if the projection is a point estimate. For multi-segment firms, the points awarded are weighted by the fraction of consolidated earnings or sales being forecast. One point multiplied by the weight as determined above is awarded for each segment forecast. One additional point is awarded if a consolidated forecast is made or if forecasts are provided for all of the firm segments and it is possible to determine the overall forecast form this information. If the forecasts are point estimates, one additional point times the appropriate weight is given. Using this procedure the maximum number of points available is 6.0 for both multi-segment and single segment firms.

Management discussion and analysis: For a single segment firm, one point is awarded for each item (18 items total) discussed provided reasons for the change is given. One additional point per item is given if the explanation includes quantitative data. e.g. market share increased $5 \%$ over last year. Maximum points $=36.0$ For multi-segment firms, the procedure is the same except for sales and operating income. For these two items a firm is given only half of the points otherwise available if the report discusses the change in consolidated sales (or operating profit) and does not discuss the change on a segment by segment basis in a manner consistent with the breakdown provided in their segmented disclosure.

Information on intangible assets: One point is awarded for each item (9 items) discussed. One additional point per item is given if the explanation includes quantitative

Social and environmental information: One point is awarded for each item (7 items) discussed. One additional point per item is given if the explanation includes quantitative 